\title{
Asymptotics of the mean-field Heisenberg model
}

\author{
Kay Kirkpatrick* and Elizabeth Meckes ${ }^{\dagger}$ \\ Department of Mathematics, University of Illinois at Urbana-Champaign \\ 1409 W. Green Street, Urbana, IL 61801, USA \\ Department of Mathematics, Case Western Reserve University \\ 220 Yost Hall, Cleveland, OH 44106, USA
}

August 25, 2021

\begin{abstract}
We consider the mean-field classical Heisenberg model and obtain detailed information about the total spin of the system by studying the model on a complete graph and sending the number of vertices to infinity. In particular, we obtain Cramér- and Sanovtype large deviations principles for the total spin and the empirical spin distribution and demonstrate a second-order phase transition in the Gibbs measures. We also study the asymptotics of the total spin throughout the phase transition using Stein's method, proving central limit theorems in the sub- and supercritical phases and a nonnormal limit theorem at the critical temperature.
\end{abstract}

\section{Introduction and summary of results}

For many models of statistical mechanics, understanding their physical behavior starts with understanding the behavior of the corresponding mean-field model-which not only suggests how the physical model behaves, but also can predict rather precisely the physical behavior in high dimensions. There are two main statistical mechanical models of ferromagnetism: the simpler and better-understood Ising model, and the more realistic and more challenging (classical) Heisenberg model, on a lattice of dimension $d$ with a spin $\sigma_{i} \in \mathbb{S}^{2}$ at each lattice site $i$, the spin configuration $\sigma \in\left(\mathbb{S}^{2}\right)^{n}$ having Hamiltonian energy in the absence of an external field (anisotropy):

$$
H_{n}(\sigma)=-\sum_{i, j} J_{i, j}\left\langle\sigma_{i}, \sigma_{j}\right\rangle .
$$

${ }^{*}$ Partially supported by NSF grants OISE-0730136 and DMS-1106770

${ }^{\dagger}$ Partially supported by the American Institute of Mathematics and NSF grant DMS-0852898 
The nearest-neighbor Heisenberg model has constant interaction $J_{i, j}=J$ for nearest neighbors $i$ and $j$, and no interaction $J_{i, j}=0$ otherwise. The mean-field version of the Heisenberg model has an averaged interaction $J_{i, j}=\frac{1}{2 n}$ for all $i, j$ and can be understood as sending the dimension $d \rightarrow \infty$ or considering the lattice to be a complete graph on $n$ vertices and sending $n \rightarrow \infty$.

The related quantum Heisenberg model has nearest-neighbor interactions of spin matrices, and in dimensions three and higher, there is a proof of a phase transition to long-range order for the antiferromagnetic quantum Heisenberg model $\left(J_{i, j}<0\right.$ for neighbors $i$ and j) [4]. Such a result has been thus far intractable for the ferromagnetic quantum Heisenberg model as well as the classical Heisenberg model, but the mean-field classical model is more amenable to rigorous analysis. In this article, we prove the existence of a second-order phase transition for the mean-field classical Heisenberg model, deriving a number of precise formulas and asymptotics for various physical quantities.

The simpler Ising model of ferromagnetism (the spins are +1 or -1 ) is better understood, and our results parallel some recent developments for the mean-field version of the Ising model, called the Curie-Weiss model. It is believed that the Curie-Weiss model accurately describes the Ising model in dimensions greater than four, in the sense that they have the same critical exponents of various physical quantities (e.g., total spin, free energy). The total spin (appropriately normalized) in the Curie-Weiss model was shown by Ellis and Newman [11] to have a Gaussian law in the non-critical regimes and law that converges to the distribution with density proportional to $e^{-x^{4} / 12}$ at the critical temperature. Recently, it was shown by Chatterjee and Shao [5] that the total spin at the critical temperature satisfies a Berry-Esseen type error bound of order $1 / \sqrt{n}$ for this non-central limit theorem. See also [3, 9] for analogous results on the Curie-Weiss-Potts model with an arbitrary finite number of discrete spins, and [2] for other related models.

There are a few results known for the Heisenberg model. In dimensions one and two, the classical Heisenberg model with nearest-neighbor interactions has no symmetry breaking, i.e., there is no phase transition to asymmetric macrostates above a critical temperature, e.g., [8]. By contrast, the classical Heisenberg model with long-range interactions or anisotropy (an external magnetic field) has a phase transition in two dimensions and higher, see [15] and references therein. And for classical, isotropic Heisenberg models with nearest-neighbor interactions in three dimensions and higher, the existence of a phase transition was shown using Fourier-based infrared bounds [13].

As for studying the mean-field classical Heisenberg model, the large-dimensional $(d \rightarrow$ $\infty)$ limit of the nearest-neighbor model on $\mathbb{Z}^{d}$, with spins in $\mathbb{S}^{2}$, has the critical inverse temperature $\beta_{c}=3$ [14]. Moreover, the large-dimensional limit is known to be a good approximation for high-dimensional models (as in Landau's theory of second order phase transitions) in the sense that below the critical temperature, the total spin is zero for all $d$, and above the critical temperature, the total spin has the correct (non-zero) limit as $d \rightarrow \infty$.

The results in this article for the isotropic classical mean-field (in the complete graph sense) Heisenberg model include:

- Large deviation principles (LDPs) for the total spin and the empirical spin distribution for each inverse temperature $\beta \in[0, \infty)$ with explicit rate functions and relative entropies. (Section 2) 
- Explicit formulas for the free energy and descriptions of the canonical macrostates and the corresponding second-order phase transition. (Section 2)

- An LDP for the empirical spin distribution with respect to the microcanonical ensemble, which has fixed energy, descriptions of the microcanonical macrostates and their second-order transition. (Section 2)

- A central limit theorem in the subcritical phase for the total spin with the usual CLT scaling $\sqrt{n}$, using Stein's method. (Section 3)

- A CLT in the supercritical phase for the total spin with a more complicated scaling, again using Stein's method. (Section 4)

- A nonnormal limit theorem for the total spin at the critical temperature, with limiting density of the squared length proportional to $t^{5} e^{-3 c t^{2}}$ (Section 5), making use of a new abstract Stein's method result for the nonnormal approximation (Appendix).

\section{The model and large deviations results}

We consider the isotropic mean-field classical Heisenberg model on a finite complete graph $G_{n}$ with $n$ vertices. That is, at each site of the graph is a spin living in $\Omega=\mathbb{S}^{2}$, so the state space is $\Omega_{n}=\left(\mathbb{S}^{2}\right)^{n}$ with $P_{n}$ the $n$-fold product of the uniform probability measure on $\mathbb{S}^{2}$. For this model, the mean-field Hamiltonian energy $H_{n}: \Omega_{n} \rightarrow \mathbb{R}$ is:

$$
H_{n}(\sigma):=-\frac{1}{2 n} \sum_{i, j=1}^{n}\left\langle\sigma_{i}, \sigma_{j}\right\rangle
$$

The energy per particle is $h_{n}(\sigma)=\frac{1}{n} H_{n}(\sigma)$, and the canonical ensemble, or Gibbs measure, is the probability measure $P_{n, \beta}$ on $\Omega_{n}$ with density (with respect to $P_{n}$ ):

$$
f(\sigma):=\frac{1}{Z} \exp \left(\frac{\beta}{2 n} \sum_{i, j=1}^{n}\left\langle\sigma_{i}, \sigma_{j}\right\rangle\right)=\frac{1}{Z} e^{-\beta H_{n}(\sigma)}=\frac{1}{Z} e^{-n \beta h_{n}(\sigma)} .
$$

Here the partition function is $Z=Z_{n}(\beta)=\int_{\Omega_{n}} \exp \left(\frac{\beta}{2 n} \sum_{i, j=1}^{n}\left\langle\sigma_{i}, \sigma_{j}\right\rangle\right) d P_{n}$.

The empirical measure $\mu_{\sigma}=\mu_{n, \sigma}$ of the spins $\left\{\sigma_{i}\right\}$ is defined to be the random measure $\mu_{n, \sigma}:=\frac{1}{n} \sum_{i=1}^{n} \delta_{\sigma_{i}}$ on $\mathbb{S}^{2}$. An interesting physical quantity is the total empirical spin, defined by

$$
S_{n}(\sigma):=n \int x d \mu_{\sigma}(x)=\sum_{i=1}^{n} \sigma_{i}
$$

For a probability measure $\nu$ on $\mathbb{S}^{2}$, define the relative entropy of $\nu$ with respect to the uniform probability measure $\mu$ by

$$
H(\nu \mid \mu):= \begin{cases}\int_{\mathbb{S}^{2}} f \log (f) d \mu & \text { if } f:=\frac{d \nu}{d \mu} \text { exists } \\ \infty & \text { otherwise }\end{cases}
$$


Let $M_{1}\left(\mathbb{S}^{2}\right)$ denote the probability measures on $\mathbb{S}^{2}$ with the weak-* topology, and also define:

$$
z_{n}(\beta):=\int_{\Omega_{n}} e^{-\beta h_{n}} d P_{n} \quad \text { and } \quad p_{n, \beta}(B):=\frac{1}{z_{n}(\beta)} \int_{B} e^{-\beta h_{n}} d P_{n} .
$$

A question of significant interest is the behavior of the total spin as a function of the inverse temperature $\beta$ in the Gibbs measures, so we begin by stating large deviations principles (LDP) for the $\mu_{n, \sigma}$, first a proposition for the noninteracting case $\beta=0$, then a theorem for general $\beta$, followed by an alternative formula for the free energy. The proposition is simply a particular instance of Sanov's theorem (see Theorem 6.2.10 of [7]).

Proposition 1. For $P_{n}$ the $n$-fold product of uniform measure on $\mathbb{S}^{2}$ and $\mu_{n, \sigma}=\frac{1}{n} \sum_{i=1}^{n} \delta_{\sigma_{i}}$ as above, if $\Gamma$ a Borel subset of $M_{1}\left(\mathbb{S}^{2}\right)$,

$$
-\inf _{\nu \in \Gamma^{\circ}} H(\nu \mid \mu) \leq \liminf _{n \rightarrow \infty} \frac{1}{n} \log P_{n}\left[\mu_{n, \sigma} \in \Gamma\right] \leq \limsup _{n \rightarrow \infty} \frac{1}{n} \log P_{n}\left[\mu_{n, \sigma} \in \Gamma\right] \leq-\inf _{\nu \in \bar{\Gamma}} H(\nu \mid \mu) ;
$$

that is, the random measures $\mu_{n, \sigma}$ satisfy an LDP with rate function $H(\cdot \mid \mu)$.

In particular, this says that the canonical macrostates $\mathcal{E}_{0}:=\{\nu: H(\nu \mid \mu)=0\}$ at $\beta=0$ are disordered since the rate function $H(\cdot \mid \mu)$ achieves its minimum of 0 for the uniform measure $\mu$ only. The positive $\beta$ canonical macrostates are $\varepsilon_{\beta}:=\left\{\nu: I_{\beta}(\nu)=0\right\}$, with $I_{\beta}$ defined below. The following theorem identifies them abstractly; Theorem [7] below describes them concretely.

Theorem 2. With notation as above, the $\mu_{n, \sigma}$ satisfy an $L D P$ on $M_{1}\left(\mathbb{S}^{2}\right)$ with rate function

$$
I_{\beta}(\nu):=H(\nu \mid \mu)-\frac{\beta}{2}\left|\int_{\mathbb{S}^{2}} x d \nu(x)\right|^{2}-\varphi(\beta),
$$

where the free energy $\varphi(\beta):=-\lim _{n \rightarrow \infty} \frac{1}{n} \log z_{n}(n \beta)=-\lim _{n \rightarrow \infty} \frac{1}{n} \log Z_{n}(\beta)$ exists and is given by

$$
\varphi(\beta)=\inf _{\nu \in M_{1}\left(\mathbb{S}^{2}\right)}\left[H(\nu \mid \mu)-\frac{\beta}{2}\left|\int_{\mathbb{S}^{2}} x d \nu(x)\right|^{2}\right] .
$$

Moreover, for every $\beta>0$, every subsequence of $P_{n, \beta}\left[\mu_{n, \sigma} \in \cdot\right]$ has a further subsequence converging weakly to $\Pi_{\beta}$, a probability measure on $M_{1}\left(\mathbb{S}^{2}\right)$ concentrated on $\mathcal{E}_{\beta}$, i.e., $\Pi_{\beta}\left(\mathcal{E}_{\beta}^{c}\right)=$ 0 . In the case that $\mathcal{E}_{\beta}=\{\nu\}$ for one $\nu \in M_{1}\left(\mathbb{S}^{2}\right)$, then the whole sequence converges weakly to $\delta_{\nu}$.

The proof of Theorem 2 makes use of an argument due to Ellis, Haven and Turkington (see Theorems 2.4 and 2.5 of [10]) in order to obtain an LDP for the empirical spins $\mu_{n, \sigma}$ in the general case of $\beta>0$, based on the result for the $\beta=0$ (independent) case.

The analysis in the appendix leads to the following.

Theorem 3. The free energy $\varphi$ has the formula (see Lemmas [21 and 22 in the Appendix):

$$
\varphi(\beta)= \begin{cases}0, & \text { if } \beta<3, \\ \Phi_{\beta}\left(g^{-1}(\beta)\right), & \text { if } \beta \geq 3,\end{cases}
$$


where $\Phi_{\beta}$ is defined by

$$
\Phi_{\beta}(k):=\log \left(\frac{k}{\sinh (k)}\right)+k \operatorname{coth}(k)-1-\frac{\beta}{2}\left(\operatorname{coth}(k)-\frac{1}{k}\right)^{2}
$$

and

$$
g(x):=\frac{x}{\operatorname{coth} x-1 / x}=\beta .
$$

In particular, the function $\varphi$ and its derivative $\varphi^{\prime}$ are continuous at the critical threshold $\beta=3$, so the phase transition is continuous, or second-order.

We remark that the critical value of $\beta=3$ identified above agrees with the largedimensional limit of Kesten and Schonmann [14].

As a corollary of the Sanov theorem for the noninteracting case $\beta=0$, or independently, as a Cramér theorem for random vectors on the sphere, one can prove the following Cramértype LDP for the total spin $M_{n}:=\frac{1}{n} \sum_{i=1}^{n} \sigma_{i}$.

Corollary 4. Let $\left\{\sigma_{i}\right\}_{i=1}^{n}$ be i.i.d. uniform random points on $\mathbb{S}^{2} \subseteq \mathbb{R}^{3}$. The total spins $M_{n}$ satisfy an LDP with rate function $I$ :

$$
P_{n}\left(M_{n} \simeq x\right) \simeq e^{-n I(x)},
$$

where $I(x)=c|x|-\log \left(\frac{\sinh (c)}{c}\right)=c \operatorname{coth}(c)-1+\log \left(\frac{c}{\sinh (c)}\right)$ and $c$ is defined by $\operatorname{coth}(c)-\frac{1}{c}=$ $|x|$.

\section{Remarks:}

(a) The function $g(y)=\operatorname{coth}(y)-\frac{1}{y}$ is strictly increasing on $(0, \infty)$, so that the equation above does uniquely define $c$ as a function of $|x|$.

(b) Readers familiar with Cramér's theorem may expect to see the rate function simply identified abstractly as the Legendre-Fenchel transform of the uniform measure on the unit sphere. The explicit rate function $I(x)$ above is indeed the Legendre-Fenchel transform of the uniform measure on the sphere, although it takes some computation to verify this. A proof of the formula as a consequence of Proposition 1 together with further analysis of the relative entropy is sketched below, immediately after the statement of Theorem [7. See also the analysis in the appendix of the free energy and Equation (3).

This noninteracting Cramér corollary has a companion result for the interacting $\beta>0$ case, which is used in Sections 4-6.

Theorem 5. Let $P_{n, \beta}$ be the Gibbs measure defined above, and let $M_{n}=M_{n}(\sigma):=\frac{1}{n} \sum_{i=1}^{n} \sigma_{i}$. Then for a Borel set $\Gamma \in \mathbb{R}$,

$$
-\inf _{x \in \Gamma^{\circ}} I_{\beta}(x) \leq \liminf _{n \rightarrow \infty} \frac{1}{n} \log P_{n, \beta}\left[\beta M_{n} \in \Gamma\right] \leq \limsup _{n \rightarrow \infty} \frac{1}{n} \log P_{n, \beta}\left[\beta M_{n} \in \Gamma\right] \leq-\inf _{x \in \bar{\Gamma}} I_{\beta}(x)
$$


where

$$
I_{\beta}(x)=c \operatorname{coth}(c)-1-\log \left(\frac{\sinh (c)}{c}\right)-\frac{\beta}{2}\left|\operatorname{coth}(c)-\frac{1}{c}\right|^{2},
$$

and $c$ is the unique element of $\mathbb{R}^{+}$such that $|x|=\operatorname{coth}(c)-\frac{1}{c}$.

One can derive this explicit Cramér-type LDP as a special case of Theorem 2 by considering the center of mass of $\mu_{\sigma, n}$ and using the computations below. Notice also that $I_{\beta}(x)=\Phi_{\beta}(c)$ from (3), where $x$ and $c$ satisfy the above formula. This result can also be proved directly by standard methods: the case $\beta=0$ is Cramér's theorem for random vectors on the sphere with rate function $I=I_{\beta=0}$ (Corollary 44), and the general case follows from Theorems 2.4 and 2.5 of [10], similar to the proof of Theorem 2 ,

The proof of Theorem 2, obtaining the LDP for the empirical measure of the spins at positive $\beta$, proceeds by applying Theorems 2.4 and 2.5 of [10] to $z_{n}(\beta)$ and $p_{n, \beta}$, and identifying the hidden (Polish) space as $M_{1}\left(\mathbb{S}^{2}\right)$, the set of Borel probability measures on $\mathbb{S}^{2}$ equipped with the weak-* topology. The hidden process is $\left\{\mu_{n, \sigma}\right\}_{n=1}^{\infty}$ as above, which satisfies an LDP with rate function $H(\cdot \mid \mu)$. The representation in question here is of the energy per particle, rather than the Hamiltonian itself:

$$
h_{n}(\sigma)=-\frac{1}{2 n^{2}} \sum_{i, j=1}^{n}\left\langle\sigma_{i}, \sigma_{j}\right\rangle=-\frac{1}{2}\left\langle\int_{\mathbb{S}^{2}} x d \mu_{n, \sigma}(x), \int_{\mathbb{S}^{2}} x d \mu_{n, \sigma}(x)\right\rangle=-\frac{1}{2}\left|\int_{\mathbb{S}^{2}} x d \mu_{n, \sigma}(x)\right|^{2} ;
$$

we define $\tilde{h}: M_{1}\left(\mathbb{S}^{2}\right) \rightarrow \mathbb{R}$ by $\tilde{h}(\nu):=-\frac{1}{2}\left|\int_{\mathbb{S}^{2}} x d \nu(x)\right|^{2}$. Note that the expression inside the norm is simply the center of mass of the measure $\nu$.

To identify the measures in $\mathcal{E}_{\beta}$ explicitly, first observe that if $f=\frac{d \nu}{d \mu}$ then $H(\nu \mid \mu)=$ $\int f \log (f) d \mu$ depends only on the value distribution of $f$; that is, (roughly speaking) once the values that $f$ takes on the $\mu$-frequency with which they are taken on are fixed, the first term is determined. This is quite easy to see if $f$ takes on only finitely many values: suppose that $f(x)=\sum_{i=1}^{n} a_{i} \mathbb{1}_{A_{i}}(x)$ with the $a_{i}$ distinct and the $A_{i}$ pairwise disjoint. Then

$$
H(\nu \mid \mu)=\sum_{i=1}^{n} a_{i} \log \left(a_{i}\right) \mu\left(A_{i}\right)
$$

and so $H(\nu \mid \mu)$ depends only on the $a_{i}$ and the $\mu\left(A_{i}\right)$. More generally, it follows from Fubini's theorem that

$$
\int f \log (f) d \mu=\int_{0}^{\infty} \mu[f \log (f)>t] d t-\int_{0}^{\infty} \mu[f \log (f)<-t] d t .
$$

Once the value distribution of $f$ is fixed, it is then easy to see that the expression $\left|\int x d \nu(x)\right|$ is maximized for corresponding densities which are symmetric about a fixed pole and decreasing as the distance from the pole increases. Consider, then, the case that $f=\frac{d \nu}{d \mu}$, a density that is symmetric about the north pole and decreasing away from the pole. That is, $\nu_{g}$ is the measure with density $f(x, y, z)=g(z)$ which is increasing in $z$. Then

$$
\begin{aligned}
H\left(\nu_{g} \mid \mu\right) & =\frac{1}{4 \pi} \int_{0}^{2 \pi} \int_{0}^{\pi} g(\cos (\theta)) \log [g(\cos (\theta))] \sin (\theta) d \theta d \varphi \\
& =\frac{1}{2} \int_{-1}^{1} g(x) \log [g(x)] d x .
\end{aligned}
$$


By the same substitution,

$$
\int_{\mathbb{S}^{2}} v d \nu_{g}(v)=\left[\begin{array}{l}
0 \\
0 \\
1
\end{array}\right] \int_{-1}^{1} \frac{x g(x)}{2} d x
$$

The problem is thus to minimize

$$
\frac{1}{2} \int_{-1}^{1} g(x) \log [g(x)] d x-\frac{\beta}{2}\left(\int_{-1}^{1} \frac{x g(x)}{2} d x\right)^{2}
$$

for $g:[-1,1] \rightarrow \mathbb{R}_{+}$such that $\frac{1}{2} \int_{-1}^{1} g(x) d x=1$ and $g$ is increasing. Observe that

$$
\frac{1}{2} \int_{-1}^{1} g(x) \log [g(x)] d x=\frac{1}{2} \int_{-1}^{1} g(x) \log \left[\frac{g(x)}{2}\right] d x+\log (2)=-h\left(\frac{g}{2}\right)+\log (2),
$$

where $h(\varphi)$ is the (usual) entropy of the density $\varphi$.

Now fix the value of $\left|\int x d \nu(x)\right| \in[0,1]$ and minimize $\frac{1}{2} \int_{-1}^{1} g(x) \log [g(x)] d x$ over the $\nu \in M_{1}\left(\mathbb{S}^{2}\right)$ corresponding to this value; this is a constrained entropy maximization problem, for which known results (see Theorem 12.1.1 from [6]) imply:

Proposition 6. Consider the class of $f:[-1,1] \rightarrow \mathbb{R}_{+}$such that

- $\int_{-1}^{1} f(x) d x=1$, and

- $\left|\int_{-1}^{1} x f(x) d x\right|=c$.

Then $f^{*}(x)=k_{1} e^{k_{2} x}$ uniquely maximizes $h(f)$ over the densities satisfying these conditions.

Now, to determine $k_{1}, k_{2}$, observe that for $f^{*}$ to satisfy the first condition,

$$
1=\int_{-1}^{1} k_{1} e^{k_{2} x} d x=\frac{2 k_{1} \sinh \left(k_{2}\right)}{k_{2}}
$$

and thus

$$
k_{1}=\frac{k_{2}}{2 \sinh \left(k_{2}\right)}
$$

For the second condition,

$$
c=k_{1} \int_{-1}^{1} x e^{k_{2} x} d x=k_{1}\left[\frac{2 \cosh \left(k_{2}\right)}{k_{2}}-\frac{2 \sinh \left(k_{2}\right)}{k_{2}^{2}}\right]=\operatorname{coth}\left(k_{2}\right)-\frac{1}{k_{2}} .
$$

Take $g^{*}=2 f^{*}$; considering all $c \in[0,1]$ and requiring $g^{*}$ to be increasing corresponds to considering all $k_{2} \in[0, \infty)$. In that case, we need to minimize

$$
\begin{aligned}
& \frac{1}{2} \int_{-1}^{1} g^{*}(x) \log \left[g^{*}(x)\right] d x-\frac{\beta}{2} c^{2} \\
& \quad=\log \left(\frac{k_{2}}{\sinh \left(k_{2}\right)}\right)+k_{2} \operatorname{coth}\left(k_{2}\right)-1-\frac{\beta}{2}\left(\operatorname{coth}\left(k_{2}\right)-\frac{1}{k_{2}}\right)^{2}=: \Phi_{\beta}\left(k_{2}\right)
\end{aligned}
$$


over all $k_{2} \in[0, \infty)$. The problem has thus been reduced to a one-dimensional calculus exercise, all of whose details are carried out in Section 7.1 of the Appendix. Those calculations lead to a critical value of the inverse temperature $\beta_{c}=3$, and to the fact that the phase transition is a continuous one (2nd order in physics parlance). Below the transition, the only macrocanonical state is the uniform distribution, and then increasing $\beta$ across the critical threshold, a spherically symmetric family of distributions with a preferred direction appears. At first the direction is hardly preferred at all, but with increasing $\beta$ the preferred direction becomes more strongly preferred, so that in the zero temperature limit $\beta \rightarrow \infty$, the macrostates are point masses. More precisely, we have the following.

Theorem 7. (a) In the subcritical case, $\beta \leq 3$, the expression (4) is minimized for $k_{2}=0$, and the corresponding $k_{1}=0$, so that the minimizing function $f^{*}=1$ and hence the canonical macrostates in the subcritical case are uniform: $\mathcal{E}_{\beta}=\{\mu\}$.

(b) In the supercritical case, $\beta>3$, the minimizing $k_{2}$ for the expression (4) is the unique strictly positive solution to

$$
x=\beta\left(\operatorname{coth}(x)-\frac{1}{x}\right),
$$

which moreover has limit $\lim _{\beta \downarrow \beta_{c}} k_{2}=0$.

The macrostates $\mathcal{E}_{\beta}$ are given by $\left\{\nu_{x}\right\}_{x \in \mathbb{S}^{2}}$, where $\nu_{x}$ is the probability measure with density which is symmetric about the pole at $x$, with density $g_{x}:[-1,1] \rightarrow \mathbb{R}$ in the $x$-direction given by $2 k_{1} e^{k_{2} x}$ with $k_{2}$ as above.

The general result on constrained entropy maximization used above also gives a proof of Corollary 4 from Proposition 1$]$ as follows.

Proof of Corollary \&. Given $x_{o} \in \mathbb{R}^{3}$ and $\epsilon>0$, take the set $\Gamma$ in Proposition 1 to be $\left\{\nu \in M_{1}\left(\mathbb{S}^{2}\right):\left|\int_{\mathbb{S}^{2}} x d \nu(x)-x_{o}\right|<\epsilon\right\}$. One must then consider

$$
\inf \left\{\int f \log (f) d \mu:\left|\int_{\mathbb{S}^{2}} x f(x) d \mu(x)-x_{o}\right|<\epsilon\right\} .
$$

This is exactly the constrained entropy maximization problem addressed in Proposition 6 and the analysis which followed, from which the form of the rate function stated in Corollary 4 follows.

We conclude this section by giving a treatment of the microcanonical ensemble, in which one fixes the energy per particle. The following result gives an LDP in that case using the results of [10].

Proposition 8. Since in the i.i.d. case, the empirical measure of the spins $\mu_{n, \sigma}=\frac{1}{n} \sum \delta_{\sigma_{i}}$ satisfies an LDP with rate $H(\cdot \mid \mu)$, we have the following:

(a) The energies $\tilde{h}\left(\mu_{n, \sigma}\right)$ and $H_{n}$ satisfy LDPs with rate $J$, called the microcanonical entropy, defined for a fixed value $u$ of the energy by:

$$
J(u):=\inf \left\{H(\nu \mid \mu): \nu \in M_{1}\left(\mathbb{S}^{2}\right), \tilde{h}(\nu)=u\right\} .
$$

The free energy $\varphi$ is a Legendre-Fenchel transform: $\varphi(\beta)=\inf _{u \in \mathbb{R}}\{\beta u+J(u)\}$. 
(b) If $u \in \operatorname{dom}(J)$, define the microcanonical Gibbs measure by

$$
P_{n}^{u, r}(A):=\frac{1}{Z_{u}} \int_{A} \mathbb{1}_{\left\{H_{n} \in[u+r, u-r]\right\}} d P_{n}
$$

where $Z_{u}:=\int_{\mathbb{S}^{2}} \mathbb{1}_{\left\{H_{n} \in[u+r, u-r]\right\}} d P_{n}$. Then for $\sigma$ distributed according to $P_{n}^{u, r}, \mu_{n, \sigma}$ satisfies an LDP with microcanonical rate function

$$
I^{u}(\nu):= \begin{cases}H(\nu \mid \mu)-J(u), & \text { if }-\frac{1}{2}\langle\nu, \nu\rangle=u \\ \infty, & \text { otherwise }\end{cases}
$$

That is,

$-\inf _{\nu \in \Gamma^{\circ}} I^{u}(\nu) \leq \lim _{r \rightarrow 0} \liminf _{n \rightarrow \infty} \frac{1}{n} \log P_{n}^{u, r}\left(\mu_{n, \sigma} \in \Gamma\right) \leq \lim _{r \rightarrow 0} \limsup _{n \rightarrow \infty} \frac{1}{n} \log P_{n}^{u, r}\left(\mu_{n, \sigma} \in \Gamma\right) \leq-\inf _{\nu \in \bar{\Gamma}} I^{u}(\nu) ;$

(c) The microcanonical macrostates are

$$
\mathcal{E}^{u}:=\{\nu: H(\nu \mid \mu)=J(u), \tilde{h}(\nu)=u\} .
$$

Again, it suffices to restrict our attention to symmetric densities $\frac{d \nu}{d \mu}$, symmetric about a pole with unit vector $\hat{z}$, i.e., $\int v d \nu(v)=c \hat{z}$, because symmetrizing about the $z$-axis reduces relative entropy: Recall that $d \mu=d \mu_{z} \frac{d z}{2}$, where $\mu_{z}$ is the uniform measure on the circle of radius $\sqrt{1-z^{2}}$. Let $\tilde{f}(z):=\int f(x, y, z) d \mu_{z}(x, y)$, which is the symmetrized version of $f$ about the $z$-axis. Now, the function $g(x)=x \log (x)$ is convex, so by Jensen's inequality,

$$
\begin{aligned}
\tilde{f}(z) \log [\tilde{f}(z)]=g\left(\int f(x, y, z) d \mu_{z}(x, y)\right) & \leq \int g(f(x, y, z)) d \mu_{z}(x, y) \\
& =\int f(x, y, z) \log \left[(f(x, y, z)] d \mu_{z}(x, y) .\right.
\end{aligned}
$$

Integrating both sides with respect to $\frac{d z}{2}$ shows that $H(\tilde{\nu} \mid \mu) \leq H(\nu \mid \mu)$, where $\nu$ and $\tilde{\nu}$ are respectively the measures with densities $f$ and $\tilde{f}$.

We can compute $J$ to be

$$
J(u)=\inf \left\{H(\tilde{\nu} \mid \mu):\left(\frac{1}{2} \int_{-1}^{1} x \tilde{f}(x) d x\right)^{2}=-2 u, \frac{1}{2} \int \tilde{f}(x) d x=1\right\}
$$

and then simplify it using the previous result on maximizing entropy, with $k_{2}$ solving coth $k_{2}-$ $\frac{1}{k_{2}}=\sqrt{-2 u}$ :

$$
J(u)=\log \left(\frac{k_{2}}{\sinh k_{2}}\right)+k_{2} \sqrt{-2 u} .
$$

The microcanonical entropy is

$$
I^{u}(\tilde{\nu})=-h(\tilde{f} / 2)+\log 2-J(u), \text { if }\left(\frac{1}{2} \int_{-1}^{1} x \tilde{f}(x) d x\right)^{2}=-2 u .
$$


The domain of $J$ is $\left(-\frac{1}{2}, 0\right]$, and the microcanonical macrostates $\mathcal{E}^{u}$ consist of rotations to any direction of $\tilde{\nu}$ with density

$$
\tilde{f}=\frac{k_{2}}{\sinh k_{2}} e^{k_{2} x}, \text { where } \operatorname{coth} k_{2}-\frac{1}{k_{2}}=\sqrt{-2 u} .
$$

In particular, $\mathcal{E}^{0}=\{\mu\}$, the completely disordered phase, and for energies close to zero $-\frac{1}{2} \ll u<0$, there is the expansion $J(u) \simeq-3 u-\frac{9}{2} u^{2}+\ldots$, and $k_{2} \simeq 3 \sqrt{-2 u}$. Thus the microcanonical macrostates for small energy are $\mathcal{E}^{u}=\left\{\nu_{x}\right\}_{x \in \mathbb{S}^{2}}$, where $\nu_{x}$ is the rotation of $\tilde{\nu}$ to the $x$ direction, again a continuous transition to the ordered phase.

\section{Limit theorems for the total spin}

In each regime (subcritical, critical, supercritical), the total spin satisfies a limit theorem. For convenience, we collect these results here; the proofs are in the subsequent three sections.

In the subcritical regime, there is the following multivariate central limit theorem.

Theorem 9. For $\beta<3$, there is a constant $c_{\beta}$ depending only on $\beta$ such that for $W_{n}=$ $\sqrt{\frac{3-\beta}{n}} \sum_{i=1}^{n} \sigma_{i}$

$$
\sup _{g: M_{1}(g), M_{2}(g) \leq 1}\left|\mathbb{E} g\left(W_{n}\right)-\mathbb{E} g(Z)\right| \leq \frac{c_{\beta} \log (n)}{\sqrt{n}}
$$

where $M_{1}(g)$ is the Lipschitz constant of $g, M_{2}(g)$ is the maximum operator norm of the Hessian of $g$, and $Z$ is a standard Gaussian random vector in $\mathbb{R}^{3}$.

The form of the theorem above may be slightly unfamiliar to some readers, so it seems worth noting explicitly that for random vectors $X$ and $Y$ in $\mathbb{R}^{d}$, the quantity

$$
\sup _{g: M_{1}(g), M_{2}(g) \leq 1}|\mathbb{E} g(X)-\mathbb{E} g(Y)|
$$

is a metric for the familiar topology of weak-star convergence together with convergence in mean on the space of probability measures. We have stated the result in the form above because the rate of convergence is probably sharp, up to the logarithmic factor. However, if one prefers the more usual $L_{1}$-Wasserstein distance as a metric for this topology, the analysis in Section 4 yields the following rate of convergence for a multivariate limit theorem there. The $L_{1}$-Wasserstein distance has several equivalent definitions; the one most relevant to us is the following: let $X$ and $Y$ be random vectors in $\mathbb{R}^{n}$. Then the $L_{1}$-Wasserstein distance $d_{W}(X, Y)$ between $X$ and $Y$ is defined by

$$
d_{W}(X, Y)=\sup _{g: M_{1}(g) \leq 1}|\mathbb{E} g(X)-\mathbb{E} g(Y)|,
$$

where as above, $M_{1}(g)$ denotes the Lipschitz constant of $g$.

Theorem 10. For $W_{n}$ constructed as above and $Z$ a standard Gaussian random vector in $\mathbb{R}^{3}$,

where $c_{\beta}$ is a constant depending only on $\beta$.

$$
d_{W}\left(W_{n}, Z\right) \leq \frac{c_{\beta}}{n^{1 / 4}}
$$


In the ordered regime, where $\beta$ is large, the spins tend to align. Indeed, it follows from the large deviations principle for $S_{n}$ that $\left|S_{n}\right|$ is close to $\frac{k_{2} n}{\beta}$ with high probability: apply Theorem 5 with $\Gamma$ a small interval around $k_{2}$, and use the fact that $k_{2}$ is the argmin of $I_{\beta}$. It is also true that $S_{n}$ is a priori spherically symmetric, making any limiting point on the sphere of radius $\frac{k_{2} n}{\beta}$ equally likely. This makes the limiting situation in the ordered regime (and at criticality, discussed below) quite different from that of the disordered regime described above; rather than a limiting distribution for $S_{n}$ about one deterministic point (i.e., zero), one must consider the fluctuations of $S_{n}$ about a spherically symmetric family of possible limiting values. In the context of statistical mechanical models of this type (i.e., the Curie-Weiss or Curie-Weiss-Potts models), this situation has typically been treated by conditioning on the limiting direction of the total spin, and then considering the conditional fluctuations about that limit (see, e.g., [12]). Here we address this issue by treating instead the fluctuations of the squared-length of the total spin; that is, we consider the random variable

$$
W_{n}:=\sqrt{n}\left[\frac{\beta^{2}}{n^{2} k_{2}^{2}}\left|\sum_{j=1}^{n} \sigma_{j}\right|^{2}-1\right] .
$$

In Section 5 it is shown that $W_{n}$ satisfies the central limit theorem stated below. Since the distribution of the total spin $\frac{1}{n} \sum_{j=1}^{n} \sigma_{j}$ is rotationally invariant, this gives a complete picture of its asymptotic behavior without making use of conditioning.

For technical reasons, the following result is given in terms of the so-called boundedLipschitz distance between $W_{n}$ and $Z$ rather than in the Wasserstein distance; boundedLipschitz distance is a metric for the topology of weak-star convergence of probability measures. The bounded-Lipschitz distance $d_{B L}(X, Y)$ between random variables $X$ and $Y$ is defined by

$$
d_{B L}(X, Y):=\sup \left\{|\mathbb{E} h(X)-\mathbb{E} h(Y)|:\|h\|_{\infty} \leq 1, M_{1}(h) \leq 1\right\}
$$

where $\|h\|_{\infty}$ is the supremum norm of $h$ and $M_{1}(h)$ is again the Lipschitz constant of $h$. We note that the definition of bounded-Lipschitz distance is sometimes given in terms of probability measures than random variables, but the two viewpoints are of course completely equivalent since the definition above depends only on the distributions of $X$ and $Y$.

Theorem 11. Let $W_{n}$ be the recentered, renormalized norm squared of the total spin, as defined in (5). There is a constant $c_{\beta}$ depending only on $\beta>3$ such that if $Z$ is a centered Gaussian random variable with variance

$$
\sigma^{2}:=\frac{4 \beta^{2}}{\left(1-\beta g^{\prime}\left(k_{2}\right)\right) k_{2}^{2}}\left[\frac{1}{k_{2}^{2}}-\frac{1}{\sinh ^{2}\left(k_{2}\right)}\right]
$$

for $g(x)=\operatorname{coth}(x)-\frac{1}{x}$, then

$$
d_{B L}\left(W_{n}, Z\right) \leq c_{\beta}\left(\frac{\log (n)}{n}\right)^{1 / 4}
$$


In Section 6, we prove the following nonnormal limit theorem for the squared-length of the total total spin at the critical temperature $\beta=3$. Again, since the total spin is spherically symmetric, this provides the limiting picture in the critical case.

Theorem 12. At the critical temperature $\beta=3$, let $W_{n}:=\frac{c_{3}\left|S_{n}\right|^{2}}{n^{3 / 2}}$, where $c_{3}$ is such that $\mathbb{E} W_{n}=1$. Let $X$ have density

$$
p(t)= \begin{cases}\frac{1}{z} t^{5} e^{-3 c t^{2}} & t \geq 0 \\ 0 & t<0\end{cases}
$$

where $c=\frac{1}{5 c_{3}}$ and $z$ is a normalizing factor. Then there is a universal constant $C$ such that

$$
\sup _{\substack{\|h\|_{\infty} \leq 1,\left\|h^{\prime}\right\|_{\infty} \leq 1 \\\left\|h^{\prime \prime}\right\|_{\infty} \leq 1}}\left|\mathbb{E} h\left(W_{n}\right)-\mathbb{E} h(X)\right| \leq \frac{C \log (n)}{\sqrt{n}} .
$$

Note: the quantity

$$
\sup _{\substack{\|h\|_{\infty} \leq 1,\left\|h^{\prime}\right\|_{\infty} \leq 1 \\\left\|h^{\prime \prime}\right\|_{\infty} \leq 1}}|\mathbb{E} h(Y)-\mathbb{E} h(X)|
$$

is a metric for the weak-star topology on random variables, so that the result above should be viewed as a limit theorem with an explicit rate of convergence in this metric. As in the subcritical case, one can employ a standard smoothing argument to obtain a rate of convergence in a more familiar metric, in this case, the bounded-Lipschitz distance defined above in Equation (6).

Theorem 13. For $W_{n}$ and $X$ as above, there is a universal constant $C$ such that

$$
d_{B L}\left(W_{n}, X\right) \leq \frac{C \log (n)}{n^{3 / 8}}
$$

Remark: The reader may have noted that the limit theorem in the subcritical case can be formulated in the $L_{1}$-Wasserstein distance, whereas those in the critical and supercritical cases are in the bounded-Lipschitz distance, which metrizes a slightly weaker topology. This is a typical by-product of the technical differences between Stein's method in multivariate (as in the subcritical case) and univariate (as in the critical and supercritical cases) settings and does not reflect essential differences.

\section{The total spin in the subcritical phase}

In this section we give proofs of Theorems 9 and 10, giving the limit theorem for $S_{n}$ in the disordered regime.

While it is not formally necessary, we find it helpful to give a heuristic computation of the variance of the total spin $S_{n}:=\sum_{i=1}^{n} \sigma_{i}$ before proceeding with rigorous proofs. Note that each of the spins $\sigma_{i}$ has a uniform marginal distribution, because the density of the Gibbs measure is rotationally invariant. Also, $\mathbb{E}\left\langle\sigma_{i}, \sigma_{i}\right\rangle=1$ for each $i$; moreover, by the 
symmetry of the Gibbs measure, $\mathbb{E}\left\langle\sigma_{i}, \sigma_{j}\right\rangle$ is the same for every pair $i \neq j$. Now, conditional on $\left\{\sigma_{j}\right\}_{j \neq 1}$, the density of $\sigma_{1}$ with respect to uniform measure on $\mathbb{S}^{2}$ is given by

$$
\frac{1}{Z_{1}} \exp \left[\frac{\beta}{n} \sum_{j \neq 1}\left\langle\theta, \sigma_{j}\right\rangle\right]
$$

where the normalization is $Z_{1}=\int_{\mathbb{S}^{2}} \exp \left[\frac{\beta}{n} \sum_{j \neq 1}\left\langle\theta, \sigma_{j}\right\rangle\right] d \mu(\theta)$. For fixed $i \in\{1, \ldots, n\}$, let $\sigma^{(i)}:=\sum_{j \neq i} \sigma_{j}$. Note that $d \mu(\theta)=\frac{\sin \alpha}{4 \pi} d \alpha d \phi$, where $(\alpha, \phi)$ are spherical coordinates; $Z_{1}$ is therefore given by

$$
Z_{1}=\frac{1}{4 \pi} \int_{0}^{2 \pi} \int_{0}^{\pi} e^{c \cos (\alpha)} \sin (\alpha) d \alpha d \phi=\frac{\sinh (c)}{c}
$$

where $c=\frac{\beta\left|\sigma^{(1)}\right|}{n}$. Now, from the conditional density above,

$$
\begin{aligned}
\mathbb{E}\left[\sigma_{1} \mid\left\{\sigma_{j}\right\}_{j \neq 1}\right] & =\frac{1}{Z_{1}} \int_{\mathbb{S}^{2}} \theta \exp \left[\frac{\beta}{n} \sum_{j \neq 1}\left\langle\theta, \sigma_{j}\right\rangle\right] d \mu(\theta) \\
& =\frac{1}{Z_{1}} \int_{\mathbb{S}^{2}}\left\langle\theta, \frac{\sigma^{(1)}}{\left|\sigma^{(1)}\right|}\right\rangle\left(\frac{\sigma^{(1)}}{\left|\sigma^{(1)}\right|}\right) \exp \left[\frac{\beta}{n}\left\langle\theta, \sigma^{(1)}\right\rangle\right] d \mu(\theta) \\
& =\left[\frac{1}{4 \pi Z_{1}} \int_{0}^{2 \pi} \int_{0}^{\pi} \cos (\alpha) \sin (\alpha) e^{\frac{\beta\left|\sigma^{(1)}\right| \cos (\alpha)}{n}} d \alpha d \phi\right]\left(\frac{\sigma^{(1)}}{\left|\sigma^{(1)}\right|}\right) \\
& =\left[\operatorname{coth}\left(\frac{\beta\left|\sigma^{(1)}\right|}{n}\right)-\frac{n}{\beta\left|\sigma^{(1)}\right|}\right]\left(\frac{\sigma^{(1)}}{\left|\sigma^{(1)}\right|}\right) .
\end{aligned}
$$

It follows from Theorem 5 that if $\beta<3$, then $\frac{\beta\left|\sigma^{(1)}\right|}{n}=o(1)$ with probability exponentially close to one: take $\Gamma=[a, b]$ for any $a>0$, and recall (see Theorem 7) that if $\beta<3$, then $I_{\beta}(x)$ has its minimum value of zero only at $x=0$. Expanding about zero gives that for $x$ small, $\operatorname{coth}(x)-\frac{1}{x} \approx \frac{x}{3}$, and so if $\beta<3$, then

$$
\mathbb{E}\left[\sigma_{1} \mid\left\{\sigma_{j}\right\}_{j \neq 1}\right] \approx \frac{\beta \sigma^{(1)}}{3 n}=\frac{\beta}{3 n} \sum_{i \neq 1} \sigma_{i} .
$$

It follows by taking inner product of both sides with $\sigma_{2}$ followed by expectation that

$$
\mathbb{E}\left\langle\sigma_{1}, \sigma_{2}\right\rangle \approx \frac{\beta}{3 n} \mathbb{E}\left\langle\sum_{j \neq 1} \sigma_{j}, \sigma_{2}\right\rangle=\frac{\beta}{3 n}\left[1+(n-2) \mathbb{E}\left\langle\sigma_{1}, \sigma_{2}\right\rangle\right],
$$

and thus

$$
\mathbb{E}\left\langle\sigma_{1}, \sigma_{2}\right\rangle \approx \frac{\beta}{3 n-\beta(n-2)} \approx \frac{\beta}{n(3-\beta)}
$$

Finally,

$$
\mathbb{E}\left|S_{n}\right|^{2}=n \mathbb{E}\left|\sigma_{1}\right|^{2}+n(n-1) \mathbb{E}\left\langle\sigma_{1}, \sigma_{2}\right\rangle \approx \frac{3 n}{3-\beta} .
$$

Theorem 9 is proved as an application of the following abstract normal approximation theorem from [16]. 
Theorem 14. Let $\left(X, X^{\prime}\right)$ be an exchangeable pair of random vectors in $\mathbb{R}^{d}$. Let $\mathcal{F}$ be a $\sigma$-algebra with $\sigma(X) \subseteq \mathcal{F}$, and suppose that there is an invertible matrix $\Lambda$, a symmetric, positive definite matrix $\Sigma$, an $\mathcal{F}$-measureable random vector $R$ and an $\mathcal{F}$-measureable random matrix $R^{\prime}$ such that

(a)

$$
\mathbb{E}\left[X^{\prime}-X \mid \mathcal{F}\right]=-\Lambda X+R
$$

(b)

$$
\mathbb{E}\left[\left(X^{\prime}-X\right)\left(X^{\prime}-X\right)^{T} \mid \mathcal{F}\right]=2 \Lambda \Sigma+R^{\prime}
$$

Then for $g \in C^{2}\left(\mathbb{R}^{d}\right)$,

$$
\begin{aligned}
\left|\mathbb{E} g(X)-\mathbb{E} g\left(\Sigma^{1 / 2} Z\right)\right| \leq M_{1}(g) & \left\|\Lambda^{-1}\right\|_{o p}\left[\mathbb{E}|R|+\frac{1}{2}\left\|\Sigma^{-1 / 2}\right\|_{o p} \mathbb{E}\left\|R^{\prime}\right\|_{H . S .}\right] \\
& +\frac{\sqrt{2 \pi}}{24} M_{2}(g)\left\|\Sigma^{-1 / 2}\right\|_{o p}\left\|\Lambda^{-1}\right\|_{o p} \mathbb{E}\left|X^{\prime}-X\right|^{3}
\end{aligned}
$$

where $M_{1}(g)$ is the Lipschitz constant of $g$ and $M_{2}(g)$ is the maximum operator norm of $\operatorname{Hess}(g)$.

Theorem 14 is a version of Stein's method of exchangeable pairs, introduced and developed in Stein's book [19], and subsequently built upon by many researchers in many contexts. One of the great virtues of the method is that it does produce limit theorems with explicit error bounds, as we have indicated above.

The theorem itself may appear rather abstract and unmotivated; it is not obvious why such conditions should lead to Gaussian behavior. Considering the univariate case for simplicity, it may be helpful to note that if $\left(X, X^{\prime}\right)$ were jointly Gaussian random variables and also close, then they would be of the form $\left(Z_{1}, \sqrt{1-\epsilon^{2}} Z_{1}+\epsilon Z_{2}\right)$ for $Z_{1}$ and $Z_{2}$ independent Gaussian random variables and $\epsilon$ small. A quick computation shows that conditions $(a)$ and (b) would indeed hold in that case with $R=0$ and $R^{\prime}$ of order $\epsilon^{4}$. For further background on Stein's method, see [1].

In order to apply Theorem 14, one must construct an exchangeable pair $\left(W_{n}, W_{n}^{\prime}\right)$. As is frequently the case in this type of argument, the exchangeable pair will first be constructed on the level of configurations, and then descend to the total spin. Given a fixed configuration $\sigma$, construct a new configuration $\sigma^{\prime}$ by letting $I$ be distributed uniformly in $\{1, \ldots, n\}$ and replacing $\sigma_{I}$ by $\sigma_{I}^{\prime}$, distributed according to the conditional distribution of the $I$-th spin, given $\left\{\sigma_{j}: j \neq I\right\}$, and defining $\sigma_{j}^{\prime}:=\sigma_{j}$ for $j \neq I$. This procedure is called the Gibbs sampler. Then the total spin of the original configuration is $W_{n}=\sqrt{\frac{3-\beta}{n}} \sum_{i=1}^{n} \sigma_{i}$ and the total spin of the new configuration is $W_{n}^{\prime}=W_{n}\left(\sigma^{\prime}\right)=W_{n}-\sqrt{\frac{3-\beta}{n}} \sigma_{I}+\sqrt{\frac{3-\beta}{n}} \sigma_{I}^{\prime}$. The following lemma gives expressions for $R, R^{\prime}, \Sigma, \Lambda$ in the present context.

Lemma 15. For the exchangeable pair $\left(W_{n}, W_{n}^{\prime}\right)$ as constructed above and $\Lambda=\left(\frac{1-\frac{\beta}{3}}{n}\right) I d$, 
(a)

$$
\mathbb{E}\left[W_{n}^{\prime}-W_{n} \mid \sigma\right]=-\Lambda W_{n}+R
$$

where

$$
R=-\frac{\beta}{3 n^{2}} W_{n}+\frac{a}{n^{3 / 2}} \sum_{i=1}^{n}\left[\operatorname{coth}\left(\frac{\beta\left|\sigma^{(i)}\right|}{n}\right)-\frac{n}{\beta\left|\sigma^{(i)}\right|}-\frac{\beta\left|\sigma^{(i)}\right|}{3 n}\right]\left(\frac{\sigma^{(i)}}{\left|\sigma^{(i)}\right|}\right)
$$

(b)

$$
\mathbb{E}\left[\left(W_{n}^{\prime}-W_{n}\right)\left(W_{n}^{\prime}-W_{n}\right)^{T} \mid \sigma\right]=2 \Lambda R^{\prime},
$$

with

$$
\begin{aligned}
& R^{\prime}=\left(\frac{1-\frac{\beta}{3}}{n}\right) {\left[\frac{1}{n} \sum_{i=1}^{n} 3 \sigma_{i} \sigma_{i}^{T}-I d\right]-\frac{2 \beta}{3 n^{2}} W_{n} W_{n}^{T}+\frac{2 \beta}{3 n^{4}} \sum_{i=1}^{n} \sigma_{i} \sigma_{i}^{T} } \\
&+\frac{a^{2}}{n^{2}} \sum_{i=1}^{n}\left\{\left[\frac{2}{3}-\frac{2 c \operatorname{coth}(c)-2}{c^{2}}\right] P_{i}+\left[\frac{\operatorname{coth}(c)}{c}-\frac{1}{c^{2}}-\frac{1}{3}\right] P_{i}^{\perp}\right. \\
&\left.-\left[\operatorname{coth}(c)-\frac{1}{c}-\frac{c}{3}\right]\left(r_{i} \sigma_{i}^{T}+\sigma_{i} r_{i}^{T}\right)\right\} .
\end{aligned}
$$

In particular, the matrix $\Sigma$ of Theorem 14 is simply the identity.

The next lemma gives bounds for the quantities $R$ and $R^{\prime}$ identified above, from which Theorem 9 follows.

Lemma 16. For $\left(W_{n}, W_{n}^{\prime}\right)$ as constructed above and $R, R^{\prime}$ as in the previous lemma, there is a constant $c_{\beta}$ depending only on $\beta$, such that

(a) $\mathbb{E}|R| \leq \frac{c_{\beta} \log (n)}{n^{3 / 2}}$

(b) $\mathbb{E}\left\|R^{\prime}\right\|_{H . S .} \leq \frac{c_{\beta}}{n^{3 / 2}} ;$

(c) $\mathbb{E}\left|W_{n}^{\prime}-W_{n}\right|^{3} \leq \frac{c_{\beta}}{n^{3 / 2}}$.

Theorem 9 now follows immediately from Theorem 14 and Lemmas 15 and 16. Theorem 10 follows from a standard smoothing argument, in which one takes a function which is assumed only to be Lipschitz with Lipschitz constant 1 and convolves with a centered Gaussian density of variance $\frac{1}{t^{2}}$, then optimizes over $t$. Such an argument is carried out carefully in Section 3 (see in particular Corollary 3.5) of [17].

Proof of Lemma 15. For notational convenience, let $a:=\sqrt{3-\beta}$.

For part (a), using the computation at the beginning of the section one has

$$
\begin{aligned}
\mathbb{E}\left[W_{n}^{\prime}-W_{n} \mid \sigma\right] & =-\frac{a}{n^{3 / 2}} \sum_{i=1}^{n}\left[\sigma_{i}-\mathbb{E}\left[\sigma_{i} \mid\left\{\sigma_{j}\right\}_{j \neq i}\right]\right] \\
& =-\frac{1}{n} W_{n}+\frac{a}{n^{3 / 2}} \sum_{i=1}^{n}\left[\operatorname{coth}\left(\frac{\beta\left|\sigma^{(i)}\right|}{n}\right)-\frac{n}{\beta\left|\sigma^{(i)}\right|}\right]\left(\frac{\sigma^{(i)}}{\left|\sigma^{(i)}\right|}\right) .
\end{aligned}
$$


Now, since $\beta<3$, it is known that $\frac{\beta\left|\sigma^{(i)}\right|}{n}=o(1)$ with probability exponentially close to 1. We therefore use the expansion of $\operatorname{coth}(x)-\frac{1}{x}$ near zero to write

$$
\begin{aligned}
\mathbb{E}\left[W_{n}^{\prime}-W_{n} \mid \sigma\right]=-\frac{1}{n} W_{n}+ & \left(\frac{a}{3 n^{5 / 2}} \sum_{i=1}^{n} \sum_{j \neq i} \beta \sigma_{j}\right) \\
& +\frac{a}{n^{3 / 2}} \sum_{i=1}^{n}\left[\operatorname{coth}\left(\frac{\beta\left|\sigma^{(i)}\right|}{n}\right)-\frac{n}{\beta\left|\sigma^{(i)}\right|}-\frac{\beta\left|\sigma^{(i)}\right|}{3 n}\right]\left(\frac{\sigma^{(i)}}{\mid \sigma^{(i) \mid}}\right) .
\end{aligned}
$$

Note that

$$
\frac{a}{3 n^{5 / 2}} \sum_{i=1}^{n} \sum_{j \neq i} \beta \sigma_{j}=\frac{1}{3 n^{2}} \sum_{i=1}^{n}\left(\beta W_{n}-\frac{a \beta \sigma_{i}}{\sqrt{n}}\right)=\frac{1}{n}\left(\frac{\beta}{3}-\frac{\beta}{3 n}\right) W_{n}
$$

The matrix $\Lambda$ of Theorem 14 is thus $\frac{1-\frac{\beta}{3}}{n} I d$ and

$$
R=-\frac{\beta}{3 n^{2}} W_{n}+\frac{a}{n^{3 / 2}} \sum_{i=1}^{n}\left[\operatorname{coth}\left(\frac{\beta\left|\sigma^{(i)}\right|}{n}\right)-\frac{n}{\beta\left|\sigma^{(i)}\right|}-\frac{\beta\left|\sigma^{(i)}\right|}{3 n}\right]\left(\frac{\sigma^{(i)}}{\left|\sigma^{(i)}\right|}\right) .
$$

We now proceed to the proof of part (b). By the same considerations as above,

$$
\begin{aligned}
\mathbb{E}\left[\left(W_{n}^{\prime}-W_{n}\right)\left(W_{n}^{\prime}-W_{n}\right)^{T} \mid \sigma\right] \\
\quad=\frac{a^{2}}{n^{2}} \sum_{i=1}^{n} \frac{1}{Z_{i}} \int_{\mathbb{S}^{2}}\left(\theta-\sigma_{i}\right)\left(\theta-\sigma_{i}\right)^{T} \exp \left[\frac{\beta}{n} \sum_{j \neq i}\left\langle\sigma_{j}, \theta\right\rangle\right] d \mu(\theta) \\
\quad=\frac{a^{2}}{n^{2}} \sum_{i=1}^{n} \frac{1}{Z_{i}} \int_{\mathbb{S}^{2}}\left[\theta \theta^{T}-\sigma_{i} \theta^{T}-\theta \sigma_{i}^{T}+\sigma_{i} \sigma_{i}^{T}\right] \exp \left[\frac{\beta}{n} \sum_{j \neq i}\left\langle\sigma_{j}, \theta\right\rangle\right] d \mu(\theta) .
\end{aligned}
$$

Letting $\theta=\theta_{1}+\theta_{2}$, where $\theta_{1}$ is the projection of $\theta$ onto the direction of $\sigma^{(i)}$ and $\theta_{2}$ is the orthogonal complement, the first term of the $i^{\text {th }}$ summand is

$$
\frac{1}{Z_{i}} \int_{\mathbb{S}^{2}}\left[\theta \theta^{T}\right] \exp \left[\frac{\beta}{n}\left\langle\sigma^{(i)}, \theta\right\rangle\right] d \mu(\theta)=\frac{1}{Z_{i}} \int_{\mathbb{S}^{2}}\left[\theta_{1} \theta_{1}^{T}+\theta_{2} \theta_{2}^{T}\right] \exp \left[\frac{\beta}{n}\left\langle\sigma^{(i)}, \theta\right\rangle\right] d \mu(\theta),
$$

since the cross terms vanish by symmetry. To compute it, write $r_{i}=\frac{\sigma^{(i)}}{\left|\sigma^{(i)}\right|}$, so that $\theta_{1}=$ $\left\langle\theta, r_{i}\right\rangle r_{i}$, and $\theta_{1} \theta_{1}^{T}=\left|\left\langle\theta, r_{i}\right\rangle\right|^{2} r_{i} r_{i}^{T}$; setting $c:=\frac{\beta \mid \sigma^{(i)}}{n}$,

$$
\frac{1}{Z_{i}} \int_{\mathbb{S}^{2}} \theta_{1} \theta_{1}^{T} \exp \left[\frac{\beta}{n}\left\langle\sigma^{(i)}, \theta\right\rangle\right] d \mu(\theta)=\frac{1}{Z_{i}}\left(\int_{\mathbb{S}^{2}}\left|\left\langle\theta, r_{i}\right\rangle\right|^{2} \exp \left[c\left\langle r_{i}, \theta\right\rangle\right] d \mu(\theta)\right) r_{i} r_{i}^{T} .
$$

(Recall that $r_{i} r_{i}^{T}$ is orthogonal projection onto the span of $r_{i}$ in $\mathbb{R}^{3}$.) In spherical coordinates (with $r_{i}$ playing the role of the north pole), the integral is then given by

$$
\frac{1}{4 \pi Z_{i}} \int_{0}^{2 \pi} \int_{0}^{\pi}|\cos \alpha|^{2} \exp [c \cos \alpha] \sin \alpha d \alpha d \phi
$$


Evaluating and using the established formula for $Z_{i}$ yields

$$
\frac{1}{Z_{i}} \int_{\mathbb{S}^{2}} \theta_{1} \theta_{1}^{T} \exp \left[\frac{1}{n}\left\langle\sigma^{(i)}, \theta\right\rangle\right] d \mu(\theta)=\frac{c^{2}-2 c \operatorname{coth}(c)+2}{c^{2}} P_{i},
$$

where $P_{i}$ is orthogonal projection onto $r_{i}$.

Now, for the second half of (8) , let $\left(\theta_{x}, \theta_{y}\right)$ be a representation of $\theta_{2}$ in orthonormal coordinates within $r_{1}^{\perp}$. Note that

$$
\int_{\mathbb{S}^{2}} \theta_{x} \theta_{y} e^{c\left\langle r_{i}, \theta\right\rangle} d \mu(\theta)=0
$$

by symmetry. Expanding in polar coordinates,

$$
\begin{aligned}
\int_{\mathbb{S}^{2}} \theta_{y}^{2} e^{c\left\langle r_{i}, \theta\right\rangle} d \mu(\theta) & =\frac{1}{4 \pi} \int_{0}^{2 \pi} \int_{0}^{\pi} \sin (\alpha)^{3} \cos (\phi)^{2} e^{c \cos (\alpha)} d \alpha d \phi \\
& =\frac{1}{4} \int_{0}^{\pi} \sin (\alpha)^{3} e^{c \cos (\alpha)} d \alpha \\
& =\frac{c \cosh (c)-\sinh (c)}{c^{3}} .
\end{aligned}
$$

Of course, the value is the same when $\theta_{y}^{2}$ is replaced by $\theta_{x}^{2}$, and thus

$$
\begin{aligned}
\frac{1}{Z_{i}} \int_{\mathbb{S}^{2}} \theta_{2} \theta_{2}^{T} \exp \left[\frac{\beta}{n}\left\langle\sigma^{(i)}, \theta\right\rangle\right] d \mu(\theta) & =\frac{c \cosh (c)-\sinh (c)}{Z_{i} c^{3}} P_{i}^{\perp} \\
& =\left[\frac{\operatorname{coth}(c)}{c}-\frac{1}{c^{2}}\right] P_{i}^{\perp},
\end{aligned}
$$

where $P_{i}^{\perp}$ is the orthogonal projection onto $r_{1}^{\perp}$.

Formulae for the middle terms follow from the computations above:

$$
\frac{1}{Z_{i}} \int_{\mathbb{S}^{2}} \theta \sigma_{i}^{T} \exp \left[\frac{\beta}{n}\left\langle\sigma^{(i)}, \theta\right\rangle\right] d \mu(\theta)=\left[\operatorname{coth}(c)-\frac{1}{c}\right] r_{i} \sigma_{i}^{T} .
$$

The next term is just the transpose of this one.

Finally, the last term is trivial:

$$
\frac{1}{Z_{i}} \int_{\mathbb{S}^{2}} \sigma_{i} \sigma_{i}^{T} \exp \left[\frac{\beta}{n}\left\langle\sigma^{(i)}, \theta\right\rangle\right] d \mu(\theta)=\sigma_{i} \sigma_{i}^{T} .
$$

Collecting terms,

$$
\begin{array}{r}
\mathbb{E}\left[\left(W_{n}^{\prime}-W_{n}\right)\left(W_{n}^{\prime}-W_{n}\right)^{T} \mid \sigma\right]=\frac{a^{2}}{n^{2}} \sum_{i=1}^{n}\left\{\left[1-\frac{2 c \operatorname{coth}(c)-2}{c^{2}}\right] P_{i}+\left[\frac{\operatorname{coth}(c)}{c}-\frac{1}{c^{2}}\right] P_{i}^{\perp}\right. \\
\left.-\left[\operatorname{coth}(c)-\frac{1}{c}\right]\left(r_{i} \sigma_{i}^{T}+\sigma_{i} r_{i}^{T}\right)+\sigma_{i} \sigma_{i}^{T}\right\},
\end{array}
$$


where $c=\frac{\beta\left|\sigma^{(i)}\right|}{n}$. Recall that if $c=o(1)$ then $\operatorname{coth}(c)-\frac{1}{c} \approx \frac{c}{3}$. In this case,

$$
\begin{aligned}
\mathbb{E}\left[\left(W_{n}^{\prime}-W_{n}\right)\left(W_{n}^{\prime}-W_{n}\right)^{T} \mid \sigma\right] & =\frac{a^{2}}{n^{2}} \sum_{i=1}^{n}\left\{\frac{1}{3} P_{i}+\frac{1}{3} P_{i}^{\perp}-\frac{c}{3}\left(r_{i} \sigma_{i}^{T}+\sigma_{i} r_{i}^{T}\right)+\sigma_{i} \sigma_{i}^{T}\right\}+R^{\prime \prime} \\
& =\frac{a^{2}}{3 n} I d+\frac{a^{2}}{n^{2}} \sum_{i=1}^{n} \sigma_{i} \sigma_{i}^{T}-\frac{a^{2} c}{3 n^{2}} \sum_{i=1}^{n}\left(r_{i} \sigma_{i}^{T}+\sigma_{i} r_{i}^{T}\right)+R^{\prime \prime}, .
\end{aligned}
$$

where the remainder term $R^{\prime \prime}$ incurred in this approximation is

$$
\begin{aligned}
R^{\prime \prime}=\frac{a^{2}}{n^{2}} \sum_{i=1}^{n}\left\{\left[\frac{2}{3}-\frac{2 c \operatorname{coth}(c)-2}{c^{2}}\right]\right. & P_{i}+\left[\frac{\operatorname{coth}(c)}{c}-\frac{1}{c^{2}}-\frac{1}{3}\right] P_{i}^{\perp} \\
& \left.-\left[\operatorname{coth}(c)-\frac{1}{c}-\frac{c}{3}\right]\left(r_{i} \sigma_{i}^{T}+\sigma_{i} r_{i}^{T}\right)\right\} .
\end{aligned}
$$

Observe further that, for the second-last term of (9), using $r_{i}=\frac{\sigma^{(i)}}{\left|\sigma^{(i)}\right|}$ and $c=\frac{\beta\left|\sigma^{(i)}\right|}{n}$ yields

$$
\frac{a^{2} c}{3 n^{2}} \sum_{i=1}^{n}\left(r_{i} \sigma_{i}^{T}+\sigma_{i} r_{i}^{T}\right)=\frac{a^{2} \beta}{3 n^{3}} \sum_{i=1}^{n} \sum_{j \neq i}\left(\sigma_{j} \sigma_{i}^{T}+\sigma_{i} \sigma_{j}^{T}\right)=\frac{2 \beta}{3 n^{2}} W_{n} W_{n}^{T}-\frac{2 a^{2} \beta}{3 n^{3}} \sum_{i=1}^{n} \sigma_{i} \sigma_{i}^{T} .
$$

Putting the pieces together,

$$
\begin{aligned}
\mathbb{E}\left[\left(W_{n}^{\prime}-W_{n}\right)\left(W_{n}^{\prime}-W_{n}\right)^{T} \mid \sigma\right] \\
=\left(\frac{3-\beta}{3 n}\right)\left[I d+\frac{1}{n} \sum_{i=1}^{n} 3 \sigma_{i} \sigma_{i}^{T}\right]-\frac{2 \beta}{3 n^{2}} W_{n} W_{n}^{T}+\frac{2 \beta}{3 n^{4}} \sum_{i=1}^{n} \sigma_{i} \sigma_{i}^{T}+R^{\prime \prime} .
\end{aligned}
$$

Note that if $X$ is uniformly distributed on the sphere, then if $U \in \mathcal{O}_{3}$,

$$
\mathbb{E}\left[X X^{T}\right]=\mathbb{E}\left[U X U^{T} U X^{T} U^{T}\right]=U \mathbb{E}\left[X X^{T}\right] U^{T},
$$

and thus $\mathbb{E}\left[X X^{T}\right]$ is a scalar matrix. Moreover, $\operatorname{Tr}\left(X X^{T}\right)=1$ since $X X^{T}$ is a rankone projection, and thus $\mathbb{E}\left[\operatorname{Tr}\left(X X^{T}\right)\right]=1$ and so since $\mathbb{E}\left[X X^{T}\right]$ is scalar, it follows that $\mathbb{E}\left[X X^{T}\right]=\frac{1}{3} I d$. That is, $\mathbb{E}\left[\sigma_{i} \sigma_{i}^{T}\right]=\frac{1}{3} I d$ for each $i$. We therefore write

$$
\mathbb{E}\left[\left(W_{n}^{\prime}-W_{n}\right)\left(W_{n}^{\prime}-W_{n}\right)^{T} \mid \sigma\right]=2\left(\frac{1-\frac{\beta}{3}}{n}\right) \Sigma+R^{\prime}
$$

as in Theorem 14, with $\Sigma=I d$ and

$$
R^{\prime}=\left(\frac{1-\frac{\beta}{3}}{n}\right)\left[\frac{1}{n} \sum_{i=1}^{n} 3 \sigma_{i} \sigma_{i}^{T}-I d\right]-\frac{2 \beta}{3 n^{2}} W_{n} W_{n}^{T}+\frac{2 \beta}{3 n^{4}} \sum_{i=1}^{n} \sigma_{i} \sigma_{i}^{T}+R^{\prime \prime} .
$$

Note in particular that the expected value of the first term of $R^{\prime}$ is zero. 
Proof of Lemma 16. Recall that $\Lambda=\left(\frac{1-\frac{\beta}{3}}{n}\right) I d$, and thus $\left\|\Lambda^{-1}\right\|_{o p}=\frac{n}{1-\frac{\beta}{3}}$.

Now, from Lemma 15.

$$
R=-\frac{\beta}{3 n^{2}} W_{n}+\frac{a}{n^{3 / 2}} \sum_{i=1}^{n}\left[\operatorname{coth}\left(\frac{\beta\left|\sigma^{(i)}\right|}{n}\right)-\frac{n}{\beta\left|\sigma^{(i)}\right|}-\frac{\beta\left|\sigma^{(i)}\right|}{3 n}\right]\left(\frac{\sigma^{(i)}}{\left|\sigma^{(i)}\right|}\right) .
$$

Note that, while it was previously argued heuristically that $\mathbb{E}\left|W_{n}\right|^{2} \approx 3$, one can in fact use the same argument together with the fact that $\operatorname{coth}(x)-\frac{1}{x} \leq \frac{x}{3}$ (shown in the proof of Lemma 21) to show that $\mathbb{E}\left|W_{n}\right|^{2} \leq 3$. It then follows that

$$
\frac{\beta}{3 n^{2}} \mathbb{E}\left|W_{n}\right| \leq \frac{\beta}{3 n^{2}} \sqrt{\mathbb{E}\left|W_{n}\right|^{2}} \leq \frac{\beta}{\sqrt{3} n^{2}} .
$$

To estimate the second half of $R$, fix $\epsilon=\epsilon(n) \in(0,1)$ to be chosen later. For notational convenience, let $r(t):=\operatorname{coth}(t)-\frac{1}{t}-\frac{t}{3}$; observe that if $t \leq \epsilon$ then $|r(t)|<b \epsilon^{2}$, where $b$ is a universal constant. Then the second half of $R$ can be estimated as

$$
\begin{aligned}
& \frac{a}{n^{3 / 2}}\left|\sum_{i=1}^{n}\left[r\left(\frac{\beta\left|\sigma^{(i)}\right|}{n}\right)\right]\left(\frac{\sigma^{(i)}}{\left|\sigma^{(i)}\right|}\right)\right| \leq \frac{a}{n^{3 / 2}}\left|\sum_{i=1}^{n}\left[r\left(\frac{\beta\left|\sigma^{(i)}\right|}{n}\right)\right]\left(\frac{\sigma^{(i)}}{\left|\sigma^{(i)}\right|}\right) \mathbb{1}\left(\frac{\beta\left|\sigma^{(i)}\right|}{n} \leq \epsilon\right)\right| \\
& +\frac{a}{n^{3 / 2}}\left|\sum_{i=1}^{n}\left[r\left(\frac{\beta\left|\sigma^{(i)}\right|}{n}\right)\right]\left(\frac{\sigma^{(i)}}{\left|\sigma^{(i)}\right|}\right) \mathbb{1}\left(\frac{\beta\left|\sigma^{(i)}\right|}{n}>\epsilon\right)\right| \\
& \leq \frac{b a \epsilon^{2}}{\sqrt{n}}+\frac{a}{n^{3 / 2}} \sum_{i=1}^{n} \mathbb{1}\left(\frac{\beta\left|\sigma^{(i)}\right|}{n}>\epsilon\right)
\end{aligned}
$$

making use of the fact that $\left|r\left(\frac{\beta\left|\sigma^{(i)}\right|}{n}\right)\right| \leq 1$ for any configuration $\sigma$. From the LDP for $\sigma^{(i)}$ (i.e., Theorem 5),

$$
\mathbb{P}\left[\frac{\beta\left|\sigma^{(i)}\right|}{n}>\epsilon\right] \leq C \exp \left[-\frac{n}{2} \inf \left\{I_{\beta}(x): x \geq \epsilon\right\}\right]
$$

where

$$
I_{\beta}(x)=c \operatorname{coth}(c)-1-\log \left(\frac{\sinh (c)}{c}\right)-\frac{\beta}{2}\left|\operatorname{coth}(c)-\frac{1}{c}\right|^{2},
$$

and $c$ is the unique element of $\mathbb{R}^{+}$such that $|x|=\operatorname{coth}(c)-\frac{1}{c}$. It is shown in the appendix that $I_{\beta}(x)$ is increasing for $\beta<3$, and thus $\inf \left\{I_{\beta}(x): x \geq \epsilon\right\}=I_{\beta}(\epsilon)$. Moreover, there is a universal constant $q>0$ such that for $\epsilon \in(0,1), I_{\beta}(\epsilon) \geq \frac{\epsilon^{2}}{6}\left(1-\frac{\beta}{3}\right)-q \epsilon^{3}$. It follows that

$$
\mathbb{P}\left[\frac{\beta\left|\sigma^{(i)}\right|}{n}>\epsilon\right] \leq C \exp \left[-\frac{n \epsilon^{2}}{12}\left(1-\frac{\beta}{3}\right)+n q \epsilon^{3}\right] .
$$

Choose $\epsilon=\epsilon(n)$ such that $\epsilon^{2}=\frac{12 \log (n)}{n\left(1-\frac{\beta}{3}\right)}$. Then $\mathbb{P}\left[\frac{\beta\left|\sigma^{(i)}\right|}{n}>\epsilon\right] \leq \frac{C^{\prime}}{n}$, and so it follows from from the bound in (10) that

$$
\frac{a}{n^{3 / 2}} \mathbb{E}\left|\sum_{i=1}^{n}\left[r\left(\frac{\beta\left|\sigma^{(i)}\right|}{n}\right)\right]\left(\frac{\sigma^{(i)}}{\left|\sigma^{(i)}\right|}\right)\right| \leq \frac{b a \epsilon^{2}}{\sqrt{n}}+\frac{a}{\sqrt{n}} \mathbb{P}\left[\frac{\beta\left|\sigma^{(1)}\right|}{n}>\epsilon\right] \leq \frac{c_{\beta} \log (n)}{n^{3 / 2}} .
$$


This completes the proof of part (a).

For part (b), recall from Lemma 15 that $R^{\prime}$ is given by

$$
\begin{aligned}
R^{\prime}=\left(\frac{1-\frac{\beta}{3}}{n}\right) & {\left[\frac{1}{n} \sum_{i=1}^{n} 3 \sigma_{i} \sigma_{i}^{T}-I d\right]-\frac{2 \beta}{3 n^{2}} W_{n} W_{n}^{T}+\frac{2 \beta}{3 n^{4}} \sum_{i=1}^{n} \sigma_{i} \sigma_{i}^{T} } \\
+ & \frac{a^{2}}{n^{2}} \sum_{i=1}^{n}\left\{\left[\frac{2}{3}-\frac{2 c \operatorname{coth}(c)-2}{c^{2}}\right] P_{i}+\left[\frac{\operatorname{coth}(c)}{c}-\frac{1}{c^{2}}-\frac{1}{3}\right] P_{i}^{\perp}\right. \\
- & {\left.\left[\operatorname{coth}(c)-\frac{1}{c}-\frac{c}{3}\right]\left(r_{i} \sigma_{i}^{T}+\sigma_{i} r_{i}^{T}\right)\right\} . }
\end{aligned}
$$

For $x \in \mathbb{R}^{n},\left\|x x^{T}\right\|_{H S}=|x|^{2}$, and so

$$
\mathbb{E}\left\|W_{n} W_{n}^{T}\right\|_{H S}=\mathbb{E}\left|W_{n}\right|^{2} \leq 3
$$

and

$$
\mathbb{E}\left\|\sigma_{i} \sigma_{i}^{T}\right\|_{H S}=\mathbb{E}\left|\sigma_{i}\right|^{2}=1,
$$

which quickly takes care of the middle two terms.

Estimating $\mathbb{E}\left\|\frac{1}{n} \sum_{i=1}^{n}\left(3 \sigma_{i} \sigma_{i}^{T}-I d\right)\right\|_{H S}$ is a bit more involved. First, recall that $\|A\|_{H S}=$ $\sqrt{\operatorname{Tr}\left(A A^{T}\right)}$, and so by the Cauchy-Schwarz inequality,

$$
\mathbb{E}\left\|\frac{1}{n} \sum_{i=1}^{n}\left(3 \sigma_{i} \sigma_{i}^{T}-I d\right)\right\|_{H S} \leq \frac{1}{n} \sqrt{\sum_{i, j=1}^{n} \mathbb{E} \operatorname{Tr}\left[\left(3 \sigma_{i} \sigma_{i}^{T}-I d\right)\left(3 \sigma_{j} \sigma_{j}^{T}-I d\right)\right]} .
$$

Now,

$$
\mathbb{E} \operatorname{Tr}\left[\left(3 \sigma_{i} \sigma_{i}^{T}-I d\right)^{2}\right]=\mathbb{E}\left[9 \operatorname{Tr}\left(\sigma_{i} \sigma_{i}^{T} \sigma_{i} \sigma_{i}^{T}\right)-6 \operatorname{Tr}\left(\sigma_{i} \sigma_{i}^{T}\right)+I d\right]=9 \mathbb{E}\left|\sigma_{i}\right|^{4}-6 \mathbb{E}\left|\sigma_{i}\right|^{2}+3=6 .
$$

Similarly, for $i \neq j$,

$$
\mathbb{E} \operatorname{Tr}\left[\left(3 \sigma_{i} \sigma_{i}^{T}-I d\right)\left(3 \sigma_{j} \sigma_{j}^{T}-I d\right)\right]=9 \mathbb{E}\left[\left\langle\sigma_{i}, \sigma_{j}\right\rangle^{2}\right]-3 .
$$

Observe that

$$
\begin{aligned}
\mathbb{E}\left[\left\langle\sigma_{1}, \sigma_{2}\right\rangle^{2} \mid\left\{\sigma_{i}\right\}_{i \neq 1}\right] & =\sigma_{2}^{T} \mathbb{E}\left[\sigma_{1} \sigma_{1}^{T} \mid\left\{\sigma_{i}\right\}_{i \neq 1}\right] \sigma_{2} \\
& =\sigma_{2}^{T}\left(\frac{1}{Z_{1}} \int_{\mathbb{S}^{2}} \theta \theta^{T} \exp \left[\frac{\beta}{n}\left\langle\theta, \sigma^{(1)}\right\rangle\right] d \mu(\theta)\right) \sigma_{2} \\
& =\sigma_{2}^{T}\left(\left[1-\frac{2 c \operatorname{coth}(c)-2}{c^{2}}\right] P_{i}+\left[\frac{c \operatorname{coth}(c)-1}{c^{2}}\right] P_{i}^{\perp}\right) \sigma_{2},
\end{aligned}
$$

where we have made use of the computation of expression (8) carried out earlier, and again $c=\frac{\beta\left|\sigma^{(1)}\right|}{n}, P_{i}$ denotes orthogonal projection onto the span of $\sigma^{(1)}$, and $P_{i}^{\perp}$ denotes orthogonal projection onto the orthogonal complement of the span of $\sigma^{(1)}$. Once again making use of the fact that $c=o(1)$ with high probability, and so $\operatorname{coth}(c)-\frac{1}{c} \approx \frac{c}{3}-\frac{c^{3}}{45}$,

$\mathbb{E}\left[\left\langle\sigma_{1}, \sigma_{2}\right\rangle^{2} \mid\left\{\sigma_{i}\right\}_{i \neq 1}\right] \approx \sigma_{2}^{T}\left(\frac{1}{3} I d+\frac{2 c^{2}}{45} P_{i}-\frac{c^{2}}{45} P_{i}^{\perp}\right) \sigma_{2}=\frac{1}{3}-\frac{c^{2}}{45}+\frac{c^{2}}{15} \mathbb{E}\left[\sigma_{2}^{T} \sigma_{1} \sigma_{1}^{T} \sigma_{2} \mid\left\{\sigma_{i}\right\}_{i \neq 1}\right]$. 
Taking expectation of both sides yields

$$
\mathbb{E}\left[\left\langle\sigma_{1}, \sigma_{2}\right\rangle^{2}\right] \approx \frac{1}{3}+\mathbb{E}\left[-\frac{c^{2}}{45}+\frac{c^{2}}{15}\left\langle\sigma_{1}, \sigma_{2}\right\rangle^{2}\right],
$$

so that

$$
\mathbb{E} \operatorname{Tr}\left[\left(3 \sigma_{i} \sigma_{i}^{T}-I d\right)\left(3 \sigma_{j} \sigma_{j}^{T}-I d\right)\right] \approx \mathbb{E}\left[-\frac{c^{2}}{5}+\frac{3 c^{2}}{5}\left\langle\sigma_{1}, \sigma_{2}\right\rangle^{2}\right] .
$$

The error incurred in this approximation (for each pair $i \neq j$ ) is

$$
\mathbb{E}\left|9 \sigma_{2}^{T}\left(\left[\frac{2}{3}-\frac{2 c \operatorname{coth}(c)-2}{c^{2}}-\frac{2 c^{2}}{45}\right] P_{i}+\left[\frac{c \operatorname{coth}(c)-1}{c^{2}}-\frac{1}{3}+\frac{c^{2}}{45}\right] P_{i}^{\perp}\right) \sigma_{2}\right| \leq r_{1} \mathbb{E} c^{3},
$$

where $r_{1}$ is a universal constant.

Now,

$$
\mathbb{E} c^{2}=\frac{\beta^{2}}{n^{2}} \sum_{i, j>1} \mathbb{E}\left\langle\sigma_{i}, \sigma_{j}\right\rangle \leq \frac{\beta^{2}}{n^{2}}\left[n-1+(n-1)(n-2) \frac{\beta}{n(3-\beta)}\right] \leq \frac{3 \beta^{2}}{n(3-\beta)},
$$

and one can then trivially also estimate that

$$
\mathbb{E} c^{3} \leq \frac{3 \beta^{3}}{n(3-\beta)},
$$

and so

$$
\mathbb{E}\left\|\frac{1}{n} \sum_{i=1}^{n}\left(3 \sigma_{i} \sigma_{i}^{T}-I d\right)\right\|_{H S} \leq \sqrt{\frac{6+\frac{r_{2}\left(\beta^{2}+\beta^{3}\right)}{(3-\beta)}}{n}} .
$$

The remaining term of the error $R^{\prime}$ is what was called $R^{\prime \prime}$ in the proof of Lemma 15, for which the remainder from Taylor's theorem also suffices:

$$
\begin{aligned}
\mathbb{E}\left\|R^{\prime \prime}\right\|_{H S} & \leq \frac{a^{2}}{n^{2}} \sum_{i=1}^{n} \mathbb{E}\left\{\left\|\left[\frac{2}{3}-\frac{2 c \operatorname{coth}(c)-2}{c^{2}}\right] P_{i}\right\|_{H S}+\left\|\left[\frac{\operatorname{coth}(c)}{c}-\frac{1}{c^{2}}-\frac{1}{3}\right] P_{i}^{\perp}\right\|_{H S}\right. \\
& \leq \frac{c_{1}(3-\beta) \beta}{n^{3}} \sum_{i=1}^{n} \mathbb{E}\left|\sigma^{(i)}\right| \\
& \leq \frac{c_{1} \sqrt{3(3-\beta)} \beta}{n^{3 / 2}},
\end{aligned}
$$

for a universal constant $c_{1}$, using the facts that $\left\|P_{i}\right\|_{H S},\left\|P_{i}^{\perp}\right\|_{H S}$ and $\left\|r_{i} \sigma_{i}^{T}\right\|_{H S}$ are all bounded by $\sqrt{2}$ or better and that $\mathbb{E}\left|\sigma^{(i)}\right| \leq \sqrt{\frac{3 n}{3-\beta}}$.

This completes the proof of part (b).

Finally, part (c) is trivial:

$$
\mathbb{E}\left|W_{n}^{\prime}-W_{n}\right|^{3}=\frac{a^{3}}{n^{3 / 2}} \mathbb{E}\left|\sigma_{I}^{\prime}-\sigma_{I}\right| \leq \frac{8 a^{3}}{n^{3 / 2}} .
$$




\section{The total spin in the supercritical phase}

In order to obtain a proof of Theorem 11, we apply the following version of Stein's abstract normal approximation theorem (see [19], p. 35). The formulation below is essentially due to Rinott and Rotar ([18], Thm 1.2), and is a univariate analog of Theorem 14 from the the previous section.

Theorem 17. Let $h: \mathbb{R} \rightarrow \mathbb{R}$ be bounded with bounded derivative. Suppose that $\left(W, W^{\prime}\right)$ is an exchangeable pair and let $\mathcal{F}$ be a $\sigma$-field with respect to which $W$ is measureable. Suppose further that there is $\lambda>0$ and an $\mathcal{F}$-measurable random variable $R$ such that

$$
\mathbb{E}\left[W^{\prime}-W \mid \mathcal{F}\right]=-\lambda W+R
$$

Then if $Z$ is a centered Gaussian random variable with variance $\sigma^{2}$,

$|\mathbb{E} h(W)-\mathbb{E} h(Z)| \leq \sqrt{\frac{\pi}{2}} \frac{\|h\|_{\infty} \mathbb{E}|R|}{\lambda}+2\|h\|_{\infty} \mathbb{E}\left|\sigma^{2}-\frac{1}{2 \lambda} \mathbb{E}\left[\left(W^{\prime}-W\right)^{2} \mid \mathcal{F}\right]\right|+\frac{\left\|h^{\prime}\right\|_{\infty} \mathbb{E}\left|W^{\prime}-W\right|^{3}}{4 \lambda}$

To apply Theorem 17 to $W_{n}=\sqrt{n}\left[\frac{\beta^{2}}{n^{2} k_{2}^{2}}\left|\sum_{j=1}^{n} \sigma_{j}\right|^{2}-1\right]$, we construct an exchangeable pair $\left(W_{n}, W_{n}^{\prime}\right)$ using the Gibbs sampler as before; that is, define $W_{n}^{\prime}$ by first replacing a randomly chosen spin in $\left\{\sigma_{i}\right\}$ according to its conditional distribution given the rest of the spins. The following lemma contains the bounds needed to obtain Theorem 11 from Theorem 17. with them, the proof of Theorem 11 is immediate.

Lemma 18. For $c_{\beta}$ a constant depending only on $\beta,\left(W_{n}, W_{n}^{\prime}\right)$ as constructed above, and $g(x)=\operatorname{coth}(x)-\frac{1}{x}$,

(a) for $\lambda=\frac{\left(1-\beta g^{\prime}\left(k_{2}\right)\right)}{n}$,

$$
\mathbb{E}\left[W_{n}^{\prime}-W_{n} \mid \sigma\right]=-\lambda W_{n}+R \quad \text { and } \quad \mathbb{E}|R| \leq \frac{c_{\beta} \log (n)}{n^{3 / 2}}
$$

(b) for $\sigma^{2}=\frac{4 \beta^{2}}{\left(1-\beta g^{\prime}\left(k_{2}\right)\right) k_{2}^{2}}\left[\frac{1}{k_{2}^{2}}-\frac{1}{\sinh ^{2}\left(k_{2}\right)}\right]$,

$$
\mathbb{E}\left|\sigma^{2}-\frac{1}{2 \lambda} \mathbb{E}\left[\left(W_{n}^{\prime}-W_{n}\right)^{2} \mid \sigma\right]\right| \leq \frac{c_{\beta}(\log (n))^{1 / 4}}{n^{1 / 4}}
$$

(c) $\mathbb{E}\left|W_{n}^{\prime}-W_{n}\right|^{3} \leq \frac{c_{\beta}}{n^{3 / 2}}$.

Proof. First note that it is shown in the Appendix (Lemma 23) that $\beta g^{\prime}\left(k_{2}\right)=\beta\left(\frac{1}{k_{2}^{2}}-\frac{1}{\sinh ^{2}\left(k_{2}\right)}\right)<$ 1 , so that for $\lambda$ and $\sigma^{2}$ as defined above are both strictly positive. 
Now,

$$
\begin{aligned}
\mathbb{E} & {\left[W_{n}^{\prime}-W_{n} \mid \sigma\right] } \\
& =-\frac{\beta^{2}}{n^{5 / 2} k_{2}^{2}} \sum_{i=1}^{n}\left[2 \sum_{k \neq i}\left\langle\sigma_{i}, \sigma_{k}\right\rangle-\mathbb{E}\left[2 \sum_{k \neq i}\left\langle\sigma_{i}, \sigma_{k}\right\rangle \mid\left\{\sigma_{j}\right\}_{j \neq i}\right]\right] \\
& =-\frac{2 \beta^{2}}{n^{5 / 2} k_{2}^{2}}\left(\left|\sum_{i=1}^{n} \sigma_{i}\right|^{2}-n\right)+\frac{2 \beta^{2}}{n^{5 / 2} k_{2}^{2}} \sum_{i=1}^{n}\left[\operatorname{coth}\left(\frac{\beta\left|\sigma^{(i)}\right|}{n}\right)-\frac{n}{\beta\left|\sigma^{(i)}\right|}\right]\left|\sigma^{(i)}\right| \\
& =-\frac{2}{n} W_{n}-\frac{2}{\sqrt{n}}+\frac{2 \beta^{2}}{n^{3 / 2} k_{2}^{2}}+\frac{2 \beta^{2}}{n^{5 / 2} k_{2}^{2}} \sum_{i=1}^{n}\left[\operatorname{coth}\left(\frac{\beta\left|\sigma^{(i)}\right|}{n}\right)-\frac{n}{\beta\left|\sigma^{(i)}\right|}\right]\left|\sigma^{(i)}\right| .
\end{aligned}
$$

For notational convenience, let $g(x):=\operatorname{coth}(x)-\frac{1}{x}$. The first simplification to the expression in (11) is to observe that $\left|\sigma^{(i)}\right|=\left|S_{n}-\sigma_{i}\right|$ is close to $\left|S_{n}\right|$ for each $i$; the error incurred by replacing each $\left|\sigma^{(i)}\right|$ with $\left|S_{n}\right|$ is estimated as follows. First,

$$
\left|g\left(\frac{\beta\left|\sigma^{(i)}\right|}{n}\right)-g\left(\frac{\beta\left|S_{n}\right|}{n}\right)\right| \leq\left\|g^{\prime}\right\|_{\infty}\left|\frac{\beta\left|\sigma^{(i)}\right|}{n}-\frac{\beta\left|S_{n}\right|}{n}\right| \leq \frac{\left\|g^{\prime}\right\|_{\infty} \beta}{n},
$$

since $S_{n}-\sigma^{(i)}=\sigma_{i}$, which has length 1 . It follows that

$$
\frac{2 \beta^{2}}{n^{5 / 2} k_{2}^{2}} \sum_{i=1}^{n} \mathbb{E}\left[\left|g\left(\frac{\beta\left|\sigma^{(i)}\right|}{n}\right)-g\left(\frac{\beta\left|S_{n}\right|}{n}\right)\right|\left|\sigma^{(i)}\right|\right] \leq \frac{2\left\|g^{\prime}\right\|_{\infty} \beta^{3}}{n^{3 / 2} k_{2}^{2}},
$$

since $\left|\sigma^{(i)}\right| \leq n$. Next, observe that

$$
\frac{2 \beta^{2}}{n^{5 / 2} k_{2}^{2}} \sum_{i=1}^{n} \mathbb{E}\left[\left|g\left(\frac{\beta\left|S_{n}\right|}{n}\right)\right||| \sigma^{(i)}|-| S_{n}||\right] \leq \frac{2\|g\|_{\infty} \beta^{2}}{n^{3 / 2} k_{2}^{2}}
$$

and so

$$
\begin{aligned}
\frac{2 \beta^{2}}{n^{5 / 2} k_{2}^{2}} \sum_{i=1}^{n} \mathbb{E} & {\left[\left|g\left(\frac{\beta\left|\sigma^{(i)}\right|}{n}\right)\right| \sigma^{(i)}\left|-g\left(\frac{\beta\left|S_{n}\right|}{n}\right)\right| S_{n}||\right] } \\
& \leq \frac{2 \beta^{2}}{n^{5 / 2} k_{2}^{2}} \sum_{i=1}^{n} \mathbb{E}\left[\left|g\left(\frac{\beta\left|\sigma^{(i)}\right|}{n}\right)-g\left(\frac{\beta\left|S_{n}\right|}{n}\right)\right|\left|\sigma^{(i)}\right|+\left|g\left(\frac{\beta\left|S_{n}\right|}{n}\right)\right||| \sigma^{(i)}|-| S_{n}||\right] \\
& \leq \frac{2\left\|g^{\prime}\right\|_{\infty} \beta^{3}+2\|g\|_{\infty} \beta^{2}}{n^{3 / 2} k_{2}^{2}}
\end{aligned}
$$

that is,

$$
\mathbb{E}\left[W_{n}^{\prime}-W_{n} \mid \sigma\right]=-\frac{2}{n} W_{n}-\frac{2}{\sqrt{n}}+\frac{2 \beta^{2}}{n^{3 / 2} k_{2}^{2}} g\left(\frac{\beta\left|S_{n}\right|}{n}\right)\left|S_{n}\right|+R_{1}
$$

where $\mathbb{E}\left|R_{1}\right| \leq \frac{c_{\beta}}{n^{3 / 2}}$. 
We next approximate $g\left(\frac{\beta\left|S_{n}\right|}{n}\right)$ by a first-order Taylor polynomial, making use of the LDP for $\left|S_{n}\right|$ (Theorem 5). We have that

$$
\limsup _{n \rightarrow \infty} \frac{1}{n} P_{n, \beta}\left[\left|\frac{\left|S_{n}\right|}{n}-\frac{k_{2}}{\beta}\right| \geq \epsilon\right] \leq-\inf _{|| x\left|-\frac{k_{2}}{\beta}\right| \geq \epsilon} I_{\beta}(x),
$$

where

$$
I_{\beta}(x)=\Phi_{\beta}(y)-\varphi(\beta), \quad \Phi_{\beta}(y)=y|x|+\log \left(\frac{y}{\sinh (y)}\right)-\frac{\beta}{2}|x|^{2},
$$

and $y$ is uniquely defined by

$$
\operatorname{coth}(y)-\frac{1}{y}=|x|
$$

Recall also that

$$
\varphi(\beta)=\inf _{x \geq 0} \Phi_{\beta}(y)=\frac{k_{2}^{2}}{2 \beta}+\log \left(\frac{k_{2}}{\sinh \left(k_{2}\right)}\right) .
$$

It was moreover shown in the Appendix (Lemmas 22 and 23) that $|x|=\frac{k_{2}}{\beta}$ corresponding to $y=k_{2}$ is the unique minimizing set for $\Phi_{\beta}$, and that $\Phi_{\beta}(y)$ is decreasing as a function of $|x|$ on $\left[0, \frac{k_{2}}{\beta}\right]$ and increasing on $\left[\frac{k_{2}}{\beta}, \infty\right)$. This means in particular that

$$
\inf _{|| x\left|-\frac{k_{2}}{\beta}\right| \geq \epsilon} I_{\beta}(x)=\min \left\{I_{\beta}\left(y\left(\frac{k_{2}}{\beta}+\epsilon\right)\right), I_{\beta}\left(y\left(\frac{k_{2}}{\beta}-\epsilon\right)\right)\right\},
$$

where by $I_{\beta}(y(t))$ we mean the value that $I_{\beta}(y)$ takes on for all $x$ with $|x|=t$ and $y$ defined in terms of $|x|$ as above.

Now, we know that $\Phi_{\beta}(y)$ is minimized on $|x|=\frac{k_{2}}{\beta}$, so that $\Phi_{\beta}^{\prime}\left(k_{2}\right)=0$. It is shown in the Appendix (Lemma 23) that $\Phi_{\beta}^{\prime \prime}\left(k_{2}\right)>0$, so that there is a constant $K_{\beta}$ such that

$$
\inf _{|| x\left|-\frac{k_{2}}{\beta}\right| \geq \epsilon} I_{\beta}(x) \geq K_{\beta} \epsilon^{2},
$$

and so

$$
P_{n, \beta}\left[\left|\frac{\left|S_{n}\right|}{n}-\frac{k_{2}}{\beta}\right| \geq \epsilon\right] \leq e^{-K_{\beta} n \epsilon^{2}} .
$$

Applying this estimate then yields

$$
\begin{aligned}
\mathbb{E}\left|\frac{2 \beta^{2}}{n^{3 / 2} k_{2}^{2}}\left[g\left(\frac{\beta\left|S_{n}\right|}{n}\right)-g\left(k_{2}\right)-g^{\prime}\left(k_{2}\right)\left(\frac{\beta\left|S_{n}\right|}{n}-k_{2}\right)\right]\right| S_{n}|| \\
\leq \frac{2 \beta^{2}}{n^{3 / 2} k_{2}^{2}}\left\|g^{\prime \prime}\right\|_{\infty} \epsilon^{2} \mathbb{E}|| S_{n}\left|\mathbb{1}\left(\left|\frac{\beta\left|S_{n}\right|}{n}-k_{2}\right| \leq \epsilon\right)\right| \\
\quad+\frac{2 \beta^{2}}{n^{3 / 2} k_{2}^{2}}\left[2\|g\|_{\infty}+\left\|g^{\prime}\right\|_{\infty}\left(\beta+k_{2}\right)\right] \mathbb{E}|| S_{n}\left|\mathbb{1}\left(\left|\frac{\beta\left|S_{n}\right|}{n}-k_{2}\right| \geq \epsilon\right)\right| \\
\leq \frac{2 \beta^{2}}{\sqrt{n} k_{2}^{2}}\left[C \epsilon^{2}+C^{\prime} e^{-K_{\beta} n \epsilon^{2}}\right]
\end{aligned}
$$


where we have also used the trivial estimate $\left|S_{n}\right| \leq n$ in the last line. Choosing $\epsilon^{2}=\frac{\log (n)}{K_{\beta} n}$ gives that

$$
\mathbb{E}\left|\frac{2 \beta^{2}}{n^{3 / 2} k_{2}^{2}}\left[g\left(\frac{\beta\left|S_{n}\right|}{n}\right)-g\left(k_{2}\right)-g^{\prime}\left(k_{2}\right)\left(\frac{\beta\left|S_{n}\right|}{n}-k_{2}\right)\right]\right| S_{n}|| \leq c_{\beta} \frac{\log (n)}{n^{3 / 2}},
$$

for some constant $c_{\beta}$ depending only on $\beta$.

Combining this estimate with (12) and recalling that $g\left(k_{2}\right)=\frac{k_{2}}{\beta}$ gives that

$$
\mathbb{E}\left[W_{n}^{\prime}-W_{n} \mid \sigma\right]=-\frac{2}{n} W_{n}+\frac{2}{\sqrt{n}}\left[\frac{\beta\left|S_{n}\right|}{n k_{2}}-1\right]+\frac{2 \beta^{2} g^{\prime}\left(k_{2}\right)}{n^{3 / 2} k_{2}^{2}}\left(\frac{\beta\left|S_{n}\right|}{n}-k_{2}\right)\left|S_{n}\right|+R_{2},
$$

where $\mathbb{E}\left|R_{2}\right| \leq \frac{c_{\beta} \log (n)}{n^{3 / 2}}$.

Now, observe that

$$
\left|S_{n}\right|=\frac{n k_{2}}{\beta} \sqrt{1+\frac{W_{n}}{\sqrt{n}}}=\frac{n k_{2}}{\beta}\left(1+\frac{W_{n}}{2 \sqrt{n}}+R_{3}\right),
$$

where $\left|R_{3}\right| \leq \frac{C\left|W_{n}\right|^{2}}{n}$. This means that the second term of (14) is

$$
\frac{W_{n}}{n}+\frac{2 R_{3}}{\sqrt{n}}
$$

and $\mathbb{E}\left|\frac{2 R_{3}}{\sqrt{n}}\right| \leq \frac{C}{n^{3 / 2}} \mathbb{E}\left|W_{n}\right|^{2} \leq \frac{C \log (n)}{n^{3 / 2}}$, using the LDP as above. Similarly,

$$
\left.\frac{2 \beta^{2} g^{\prime}\left(k_{2}\right)}{n^{3 / 2} k_{2}^{2}} \mathbb{E}\left|\left(\frac{\beta\left|S_{n}\right|}{n}-k_{2}\right)\right| S_{n}\left|-\frac{k_{2} W_{n}}{2 \sqrt{n}}\right| S_{n}|| \leq \frac{c_{\beta}}{n^{5 / 2}} \mathbb{E}\left[\left|W_{n}\right|^{2}\left|S_{n}\right|\right]\right] \leq \frac{c_{\beta} \log (n)}{n^{3 / 2}},
$$

and

$$
\frac{2 \beta^{2} g^{\prime}\left(k_{2}\right)}{n^{3 / 2} k_{2}^{2}} \mathbb{E}\left|\frac{k_{2} W_{n}}{2 \sqrt{n}}\right| S_{n}\left|-\frac{\sqrt{n} k_{2}^{2} W_{n}}{2 \beta}\right| \leq \frac{2 \beta^{2} g^{\prime}\left(k_{2}\right)}{n^{3 / 2} k_{2}^{2}} \mathbb{E}\left[\frac{C k_{2}^{2}\left|W_{n}\right|^{2}}{4 \beta}\right] \leq \frac{c_{\beta} \log (n)}{n^{3 / 2}}
$$

again using that $\mathbb{E}\left|W_{n}\right|^{2} \leq \log (n)$. Combining these estimates with (14) yields

$$
\mathbb{E}\left[W_{n}^{\prime}-W_{n} \mid \sigma\right]=-\frac{\left(1-\beta g^{\prime}\left(k_{2}\right)\right)}{n} W_{n}+R_{4}
$$

where again $\mathbb{E}\left|R_{4}\right| \leq \frac{c_{\beta} \log (n)}{n^{3 / 2}}$. This completes the proof of part $(a)$.

For part (b), observe that by definition,

$$
\begin{aligned}
\mathbb{E}\left[\left(W_{n}^{\prime}-W_{n}\right)^{2} \mid \sigma\right] & =\frac{\beta^{4}}{n^{4} k_{2}^{4}} \sum_{i=1}^{n} \mathbb{E}\left[\left(2 \sum_{j \neq i}\left\langle\sigma_{i}^{*}-\sigma_{i}, \sigma_{j}\right\rangle\right)^{2} \mid \sigma, I=i\right] \\
& =\frac{4 \beta^{4}}{n^{4} k_{2}^{4}} \sum_{i=1}^{n} \sum_{j, k \neq i} \mathbb{E}\left[\sigma_{j}^{T}\left(\sigma_{i}^{*}-\sigma_{i}\right)\left(\sigma_{i}^{*}-\sigma_{i}\right)^{T} \sigma_{k} \mid \sigma, I=i\right] .
\end{aligned}
$$


Now, $\mathbb{E}\left[\left(\sigma_{i}^{*}-\sigma_{i}\right)\left(\sigma_{i}^{*}-\sigma_{i}\right)^{T} \mid \sigma, I=i\right]$ was already computed exactly in the $\beta<3$ case, and was found to be

$$
\begin{aligned}
\mathbb{E}\left[\left(\sigma_{i}^{*}-\sigma_{i}\right)\left(\sigma_{i}^{*}-\sigma_{i}\right)^{T} \mid \sigma, I=i\right]=\left\{\left[1-\frac{2 c_{i} \operatorname{coth}\left(c_{i}\right)-2}{c_{i}^{2}}\right] P_{i}+\left[\frac{\operatorname{coth}\left(c_{i}\right)}{c_{i}}-\frac{1}{c_{i}^{2}}\right] P_{i}^{\perp}\right. \\
\left.-\left[\operatorname{coth}\left(c_{i}\right)-\frac{1}{c_{i}}\right]\left(r_{i} \sigma_{i}^{T}+\sigma_{i} r_{i}^{T}\right)+\sigma_{i} \sigma_{i}^{T}\right\},
\end{aligned}
$$

where $c_{i}=\frac{\beta\left|\sigma^{(i)}\right|}{n}, r_{i}=\frac{\sigma^{(i)}}{\left|\sigma^{(i)}\right|}$, and $P_{i}$ is orthogonal projection onto $r_{i}$.

Recall that

$$
P_{n, \beta}\left[\left|\frac{\beta\left|\sigma^{(i)}\right|}{n}-\frac{k_{2}(n-1)}{n}\right| \geq \epsilon\right] \leq e^{-K_{\beta} n \epsilon^{2}}
$$

and that

$$
\operatorname{coth}\left(k_{2}\right)-\frac{1}{k_{2}}=\frac{k_{2}}{\beta}
$$

Using this above,

$$
\begin{aligned}
\mathbb{E}\left[\left(\sigma_{i}^{*}-\sigma_{i}\right)\left(\sigma_{i}^{*}-\sigma_{i}\right)^{T} \mid \sigma, I=i\right] & =\left(1-\frac{2}{\beta}\right) P_{i}+\frac{1}{\beta} P_{i}^{\perp}-\frac{k_{2}}{\beta}\left(r_{i} \sigma_{i}^{T}+\sigma_{i} r_{i}^{T}\right)+\sigma_{i} \sigma_{i}^{T}+R_{i}^{\prime} \\
& =\frac{1}{\beta} I d+\left(1-\frac{3}{\beta}\right) P_{i}-\frac{k_{2}}{\beta}\left(r_{i} \sigma_{i}^{T}+\sigma_{i} r_{i}^{T}\right)+\sigma_{i} \sigma_{i}^{T}+R_{i}^{\prime},
\end{aligned}
$$

where

$$
R_{i}^{\prime}=\left(\frac{g\left(c_{i}\right)}{c_{i}}-\frac{1}{\beta}\right) I d+\left(\frac{3 g\left(c_{i}\right)}{c_{i}}-\frac{3}{\beta}\right) P_{i}-\left(g\left(c_{i}\right)-\frac{k_{2}}{\beta}\right)\left(r_{i} \sigma_{i}^{T}+\sigma_{i} r_{i}^{T}\right) .
$$

Ignoring the $R_{i}^{\prime}$ for the moment and putting the main term of (17) into (16) yields

$$
\frac{4 \beta^{4}}{n^{4} k_{2}^{4}} \sum_{i} \sum_{j, k \neq i}\left[\frac{1}{\beta}\left\langle\sigma_{j}, \sigma_{k}\right\rangle+\left(1-\frac{3}{\beta}\right) \sigma_{j}^{T} P_{i} \sigma_{k}-\frac{k_{2}}{\beta}\left(\sigma_{j}^{T} r_{i} \sigma_{i}^{T} \sigma_{k}+\sigma_{j}^{T} \sigma_{i} r_{i}^{T} \sigma_{k}\right)+\sigma_{j}^{T} \sigma_{i} \sigma_{i}^{T} \sigma_{k}\right] .
$$

The first term is

$$
\frac{4 \beta^{3}}{n^{4} k_{2}^{4}} \sum_{i} \sum_{j, k \neq i}\left\langle\sigma_{j}, \sigma_{k}\right\rangle=\frac{4 \beta^{3}}{n^{4} k_{2}^{4}} \sum_{i=1}^{n}\left|\sigma^{(i)}\right|^{2} .
$$

For the second term, note that $\sigma_{j}^{T} P_{i} \sigma_{k}=\operatorname{Tr}\left(\sigma_{k} \sigma_{j}^{T} r_{i} r_{i}^{T}\right)$, and so

$$
\sum_{j, k \neq i} \sigma_{j}^{T} P_{i} \sigma_{k}=\operatorname{Tr}\left(\sigma^{(i)}\left[\sigma^{(i)}\right]^{T} r_{i} r_{i}^{T}\right)=\left\langle\sigma^{(i)}, r_{i}\right\rangle^{2}=\left|\sigma^{(i)}\right|^{2}
$$

and thus

$$
\frac{4 \beta^{4}}{n^{4} k_{2}^{4}} \sum_{i} \sum_{j, k \neq i}\left(1-\frac{3}{\beta}\right) \sigma_{j}^{T} P_{i} \sigma_{k}=\frac{4 \beta^{4}}{n^{4} k_{2}^{4}}\left(1-\frac{3}{\beta}\right) \sum_{i}\left|\sigma^{(i)}\right|^{2}
$$


Similarly, for the first half of the third term,

$$
-\frac{4 \beta^{3}}{n^{4} k_{2}^{3}} \sum_{i} \sum_{j, k \neq i} \sigma_{j}^{T} r_{i} \sigma_{i}^{T} \sigma_{k}=-\frac{4 \beta^{3}}{n^{4} k_{2}^{3}} \sum_{i}\left|\sigma^{(i)}\right|\left\langle\sigma^{(i)}, \sigma_{i}\right\rangle,
$$

and the second half is the same. Finally,

$$
\frac{4 \beta^{4}}{n^{4} k_{2}^{4}} \sum_{i} \sum_{j, k \neq i} \sigma_{j}^{T} \sigma_{i} \sigma_{i}^{T} \sigma_{k}=\frac{4 \beta^{4}}{n^{4} k_{2}^{4}} \sum_{i}\left\langle\sigma_{i}, \sigma^{(i)}\right\rangle^{2}
$$

all together,

$$
\begin{aligned}
\mathbb{E}\left[\left(W_{n}^{\prime}-W_{n}\right)^{2} \mid \sigma\right]=\frac{4 \beta^{4}}{n^{4} k_{2}^{4}} \sum_{i} & {\left[\left(1-\frac{2}{\beta}\right)\left|\sigma^{(i)}\right|^{2}-\frac{2 k_{2}}{\beta}\left|\sigma^{(i)}\right|\left\langle\sigma_{i}, \sigma^{(i)}\right\rangle+\left\langle\sigma_{i}, \sigma^{(i)}\right\rangle^{2}\right] } \\
+ & \frac{4 \beta^{4}}{n^{4} k_{2}^{4}} \sum_{i} \sum_{j, k \neq i} \sigma_{j}^{T} R_{i}^{\prime} \sigma_{k} .
\end{aligned}
$$

In order to apply Theorem [17, one must recognize in this expression a deterministic part (which is then called $2 \lambda \sigma^{2}$, from which $\sigma^{2}$ is then determined), plus a mean zero part. With this motivation in mind, we write

$$
\begin{gathered}
\mathbb{E}\left[\left(W_{n}^{\prime}-W_{n}\right)^{2} \mid \sigma\right] \\
=\frac{4 \beta^{4}}{n^{3} k_{2}^{4}}\left[2\left(1-\frac{2}{\beta}\right) \frac{(n-1)^{2} k_{2}^{2}}{\beta^{2}}-\frac{2 n^{2} k_{2}^{4}}{\beta^{4}}\right]+\frac{4 \beta^{4}}{n^{4} k_{2}^{4}} \sum_{i}\left(1-\frac{2}{\beta}\right)\left(\left|\sigma^{(i)}\right|^{2}-\frac{(n-1)^{2} k_{2}^{2}}{\beta^{2}}\right) \\
+\frac{4 \beta^{4}}{n^{4} k_{2}^{4}} \sum_{i}\left[-\frac{2 k_{2}}{\beta}\left(\left|\sigma^{(i)}\right|\left\langle\sigma_{i}, \sigma^{(i)}\right\rangle-\frac{n^{2} k_{2}^{3}}{\beta^{3}}\right)+\left\langle\sigma_{i}, \sigma^{(i)}\right\rangle^{2}-\left(1-\frac{2}{\beta}\right) \frac{(n-1)^{2} k_{2}^{2}}{\beta^{2}}\right] \\
+\frac{4 \beta^{4}}{n^{4} k_{2}^{4}} \sum_{i} \sum_{j, k \neq i} \sigma_{j}^{T} R_{i}^{\prime} \sigma_{k},
\end{gathered}
$$

and define $\sigma$ such that, to top order in $n$,

$$
\frac{4 \beta^{4}}{n^{3} k_{2}^{4}}\left[2\left(1-\frac{2}{\beta}\right) \frac{(n-1)^{2} k_{2}^{2}}{\beta^{2}}-\frac{2 n^{2} k_{2}^{4}}{\beta^{4}}\right]=2 \lambda \sigma^{2},
$$

for $\lambda=\frac{1-\beta g^{\prime}\left(k_{2}\right)}{n}$ as above. Note that the top order in $n$ of the expression inside the parentheses can be simplified to

$$
\begin{aligned}
\frac{2 n^{2} k_{2}^{2}}{\beta^{2}}\left[1-\frac{2}{\beta}-\frac{k_{2}^{2}}{\beta^{2}}\right] & =\frac{2 n^{2} k_{2}^{2}}{\beta^{2}}\left[1-\frac{2\left(\operatorname{coth}\left(k_{2}\right)-\frac{1}{k_{2}}\right)}{k_{2}}-\left(\operatorname{coth}\left(k_{2}\right)-\frac{1}{k_{2}}\right)^{2}\right] \\
& =\frac{2 n^{2} k_{2}^{2}}{\beta^{2}}\left[1-\frac{\cosh ^{2}\left(k_{2}\right)}{\sinh ^{2}\left(k_{2}\right)}+\frac{1}{k_{2}^{2}}\right] \\
& =\frac{2 n^{2} k_{2}^{2}}{\beta^{2}}\left[\frac{1}{k_{2}^{2}}-\frac{1}{\sinh ^{2}\left(k_{2}\right)}\right]>0
\end{aligned}
$$


so defining $\sigma$ in this way does in fact yield a strictly positive value of $\sigma^{2}$ which depends only on $\beta$ and is independent of $n$.

Then to apply Theorem 17 it is necessary to estimate the expected absolute value of each of the terms above (except the $2 \lambda \sigma^{2}$ part).

For the first term, it follows as before from the LDP for $\left|\sigma^{(i)}\right|$ that

$$
\left.\mathbb{E}|| \sigma^{(i)}\right|^{2}-\frac{(n-1)^{2} k_{2}^{2}}{\beta^{2}} \mid \leq(n-1)^{2}\left[\epsilon+e^{-K_{\beta}(n-1) \epsilon^{2}}\right]
$$

taking $\epsilon=\sqrt{\frac{\log (n)}{K_{\beta}(n-1)}}$ shows that

$$
\left.\mathbb{E}|| \sigma^{(i)}\right|^{2}-\frac{(n-1)^{2} k_{2}^{2}}{\beta^{2}} \mid \leq c_{\beta} \sqrt{\log (n)} n^{3 / 2}
$$

Next, observe that

$$
\left|\left\langle\sigma_{i}, \sigma^{(i)}\right\rangle\right| \sigma^{(i)}\left|-\left\langle\sigma_{i}, S_{n}\right\rangle\right| S_{n}|| \leq\left|\left\langle\sigma_{i}, \sigma^{(i)}\right\rangle\right|+\left|S_{n}\right| \leq 2 n
$$

Moreover,

$$
\frac{4 \beta^{4}}{n^{4} k_{2}^{4}} \mathbb{E}\left|\sum_{i} \frac{2 k_{2}}{\beta}\left(\left|S_{n}\right|\left\langle\sigma_{i}, S_{n}\right\rangle-\frac{n^{2} k_{2}^{3}}{\beta^{3}}\right)\right|=\left.\frac{8 \beta^{3}}{n^{4} k^{3}} \mathbb{E}|| S_{n}\right|^{3}-\frac{n^{3} k_{2}^{3}}{\beta^{3}} \mid \leq \frac{c_{\beta} \sqrt{\log (n)}}{n^{3 / 2}} .
$$

Now, using the same definitions for $g, c_{i}, r_{i}$ and $P_{i}$ as before,

$$
\begin{aligned}
\mathbb{E}\left[\left\langle\sigma_{i}, \sigma^{(i)}\right\rangle^{2}\right] & =\mathbb{E}\left[\sum_{j, k \neq i} \sigma_{j}^{T} \mathbb{E}\left[\sigma_{i} \sigma_{i}^{T} \mid\left\{\sigma_{\ell}\right\}_{\ell \neq i}\right] \sigma_{k}\right] \\
& =\mathbb{E}\left[\sum_{j, k \neq i} \sigma_{j}^{T}\left[\left(1-\frac{3 g\left(c_{i}\right)}{c_{i}}\right) P_{i}+\frac{g\left(c_{i}\right)}{c_{i}} I d\right] \sigma_{k}\right]=\mathbb{E}\left[\left(1-\frac{2 g\left(c_{i}\right)}{c_{i}}\right)\left|\sigma^{(i)}\right|^{2}\right]
\end{aligned}
$$

this explains the choice of constants in (18). Furthermore,

$$
\begin{aligned}
\mathbb{E} & {\left[\sum_{i}\left(\left\langle\sigma_{i}, \sigma^{(i)}\right\rangle^{2}-\left(1-\frac{2}{\beta}\right) \frac{(n-1)^{2} k_{2}^{2}}{\beta^{2}}\right)\right]^{2} } \\
= & n \mathbb{E}\left(\left\langle\sigma_{1}, \sigma^{(1)}\right\rangle^{2}-\left(1-\frac{2}{\beta}\right) \frac{(n-1)^{2} k_{2}^{2}}{\beta^{2}}\right)^{2} \\
& +n(n-1) \mathbb{E}\left(\left\langle\sigma_{1}, \sigma^{(1)}\right\rangle^{2}-\left(1-\frac{2}{\beta}\right) \frac{(n-1)^{2} k_{2}^{2}}{\beta^{2}}\right)\left(\left\langle\sigma_{2}, \sigma^{(2)}\right\rangle^{2}-\left(1-\frac{2}{\beta}\right) \frac{(n-1)^{2} k_{2}^{2}}{\beta^{2}}\right) .
\end{aligned}
$$

The first term will simply be estimated by $c_{\beta} n^{5}$. For the second, first let $\sigma^{(1,2)}:=\sum_{j>2} \sigma_{j}$ and observe that

$$
\left|\mathbb{E}\left\langle\sigma_{1}, \sigma^{(1)}\right\rangle^{2}\left\langle\sigma_{2}, \sigma^{(2)}\right\rangle^{2}-\mathbb{E}\left\langle\sigma_{1}, \sigma^{(1)}\right\rangle^{2}\left\langle\sigma_{2}, \sigma^{(1,2)}\right\rangle^{2}\right| \leq C n^{3} .
$$


Now,

$$
\begin{aligned}
\mathbb{E}\left\langle\sigma_{1}, \sigma^{(1)}\right\rangle^{2}\left\langle\sigma_{2}, \sigma^{(1,2)}\right\rangle^{2} & =\mathbb{E}\left[\sum_{i, j>1} \sum_{k, \ell>2} \sigma_{i}^{T} \sigma_{1} \sigma_{1}^{T} \sigma_{j} \sigma_{k}^{T} \sigma_{2} \sigma_{2}^{T} \sigma_{\ell}\right] \\
& =\mathbb{E}\left[\sum_{i, j>1} \sum_{k, \ell>2} \sigma_{i}^{T} \mathbb{E}\left[\sigma_{1} \sigma_{1}^{T} \mid\left\{\sigma_{m}\right\}_{m>1}\right] \sigma_{j} \sigma_{k}^{T} \sigma_{2} \sigma_{2}^{T} \sigma_{\ell}\right] \\
& =\mathbb{E}\left[\left(1-\frac{2 g\left(c_{1}\right)}{c_{1}}\right)\left|\sigma^{(1)}\right|^{2}\left\langle\sigma_{2}, \sigma^{(1,2)}\right\rangle^{2}\right]
\end{aligned}
$$

By the LDP for $\sigma^{(1)}$, we can replace $\frac{g\left(c_{1}\right)}{c_{1}}$ by $\frac{1}{\beta}$ and $\left|\sigma^{(1)}\right|$ by $\frac{(n-1) k_{2}}{\beta}$, incurring an error of size $c_{\beta} \sqrt{\log (n)} n^{7 / 2}$. At this point we are left with

$$
\mathbb{E}\left[\left(1-\frac{2}{\beta}\right) \frac{(n-1)^{2} k_{2}^{2}}{\beta^{2}}\left\langle\sigma_{2}, \sigma^{(1,2)}\right\rangle^{2}\right]
$$

We can now go back to $\sigma^{(2)}$ instead of $\sigma^{(1,2)}$ (with another loss of order $n^{3}$ ), and therefore replace the last expression with

$$
\mathbb{E}\left[\left(1-\frac{2}{\beta}\right) \frac{(n-1)^{2} k_{2}^{2}}{\beta^{2}}\left(1-\frac{2 g\left(c_{2}\right)}{c_{2}}\right)\left|\sigma^{(2)}\right|^{2}\right],
$$

(using the expression for $\mathbb{E}\left[\left\langle\sigma_{i}, \sigma^{(i)}\right\rangle\right]$ obtained above). One final application of the LDP for $\sigma^{(2)}$ now means that, with loss of the same order as before, this expression is equal to

$$
\left(1-\frac{2}{\beta}\right)^{2} \frac{(n-1)^{4} k_{2}^{4}}{\beta^{4}}
$$

Using these approximations in (19) now yields

$$
\mathbb{E}\left[\sum_{i}\left(\left\langle\sigma_{i}, \sigma^{(i)}\right\rangle^{2}-\left(1-\frac{2}{\beta}\right) \frac{(n-1)^{2} k_{2}^{2}}{\beta^{2}}\right)\right]^{2} \leq c_{\beta} \sqrt{\log (n)} n^{11 / 2},
$$

and so

$$
\frac{4 \beta^{4}}{n^{4} k_{2}^{4}} \mathbb{E}\left|\sum_{i}\left(\left\langle\sigma_{i}, \sigma^{(i)}\right\rangle^{2}-\left(1-\frac{2}{\beta}\right) \frac{(n-1)^{2} k_{2}^{2}}{\beta^{2}}\right)\right| \leq c_{\beta}(\log (n))^{1 / 4} n^{-5 / 4} .
$$

Exactly the same sorts of arguments using LDP for $\sigma^{(i)}$ also imply that all the errors from the $R_{i}^{\prime}$ terms are smaller than those already accounted for.

Finally, part $(c)$ is trivial:

$$
\mathbb{E}\left|W_{n}^{\prime}-W_{n}\right|^{3}=\frac{8 \beta^{6}}{n^{9 / 2} k_{2}^{6}} \mathbb{E}\left|\sum_{j \neq I}\left\langle\sigma_{I}^{*}-\sigma_{I}, \sigma_{j}\right\rangle\right|^{3} \leq \frac{8 \beta^{6}}{n^{3 / 2} k_{2}^{6}} .
$$




\section{The critical temperature}

As in the previous section, we begin with an discussion of the correct normalization for $S_{n}$ in the current regime. Recall that the LDP for $\left|S_{n}\right|$ (Theorem 5 ) implies in particular that

$$
\mathbb{P}\left[\frac{3\left|S_{n}\right|}{n}>\epsilon\right] \leq C \exp \left[-\frac{n}{2} \inf \left\{I_{3}(x): x \geq \epsilon\right\}\right]
$$

where

$$
I_{3}(x)=c \operatorname{coth}(c)-1-\log \left(\frac{\sinh (c)}{c}\right)-\frac{3}{2}\left|\operatorname{coth}(c)-\frac{1}{c}\right|^{2},
$$

and $c$ is the unique element of $\mathbb{R}^{+}$such that $|x|=\operatorname{coth}(c)-\frac{1}{c}$. It is shown in the appendix that $I_{3}(x)$ is increasing, and thus $\inf \left\{I_{3}(x): x \geq \epsilon\right\}=I_{3}(\epsilon)$. Moreover, there is a universal constant $r>0$ such that for $\epsilon \in(0,1), I_{3}(\epsilon) \geq r \epsilon^{4}$ (note in particular the difference from the subcritical case). It follows that

$$
\mathbb{P}\left[\frac{3\left|S_{n}\right|}{n}>\epsilon\right] \leq C \exp \left[-c n \epsilon^{4}\right] .
$$

for some constant $c>0$, and so

$$
\mathbb{E}\left[\frac{3\left|S_{n}\right|}{n}\right] \leq \epsilon+3 C \exp \left[-c n \epsilon^{4}\right] .
$$

Choosing $\epsilon^{4}=\frac{\log (n)}{c n}$ shows that $\mathbb{E}\left[\left|S_{n}\right|\right] \leq \frac{C \sqrt[4]{\log (n)}}{n^{3 / 4}}$. This at least suggests what turns out to be the correct normalization for the total spin. In fact, one can use a modification of the argument given in the beginning of section 4 to show that

$$
\mathbb{E}\left|S_{n}\right|^{2}=n+\mathbb{E}\left[\left|S_{n}\right|^{2}-\frac{\left|S_{n}\right|^{4}}{5 n^{2}}-\frac{2\left|S_{n}\right|^{2}}{n}+\frac{2 \cdot 3^{5}\left|S_{n}\right|^{6}}{945 n^{4}}+\text { lower order terms }\right],
$$

from which it eventually follows that, to top order in $n$, there is a $c_{3}$ such that

$$
\mathbb{E}\left|S_{n}\right|^{2}=\frac{n^{3 / 2}}{c_{3}} \quad \mathbb{E}\left|S_{n}\right|^{4}=5 n^{3}
$$

The proof of the limit theorem for $W_{n}=\frac{C_{3}\left|S_{n}\right|^{2}}{n^{3 / 2}}$ is essentially via the so-called "density approach" to Stein's method introduced by Stein, Diaconis, Holmes and Reinert [20]; see also the recent work of Chatterjee and Shao [5], with an application to the total spin of the mean-field Ising model (i.e., the Curie-Weiss model). The following theorem provides the framework we use for the approximation; the proof is given in Section 7.2 of the Appendix.

Theorem 19. Let $\left(W, W^{\prime}\right)$ be an exchangeable pair of positive random variables. Suppose there exists a $\sigma$-field $\mathcal{F} \supseteq \sigma(W)$, $\mathcal{F}$-measurable random variables $R$ and $R^{\prime}$ and $k>0$ deterministic such that

$$
\mathbb{E}\left[W^{\prime}-W \mid \mathcal{F}\right]=3 k\left(1-c W^{2}\right)+R
$$


and

$$
\mathbb{E}\left[\left(W^{\prime}-W\right)^{2} \mid \mathcal{F}\right]=k W+R^{\prime}
$$

Let $X$ have density

$$
p(t)= \begin{cases}\frac{1}{z} x^{5} e^{-\frac{c t^{2}}{2}} & t \geq 0 \\ 0 & t<0 .\end{cases}
$$

Then there are constants $C_{1}, C_{2}, C_{3}$ depending only on $c$ such that for all $h \in C^{2}(\mathbb{R})$,

$$
\begin{aligned}
|\mathbb{E} h(W)-\mathbb{E} h(X)| \leq & \frac{C_{1}\|h\|_{\infty}}{k} \mathbb{E}|R|+\left(\frac{C_{2}\left(\|h\|_{\infty}+\left\|h^{\prime}\right\|_{\infty}\right)}{k}\right) \mathbb{E}\left|R^{\prime}\right| \\
& +\left(\frac{C_{3}\left(\|h\|_{\infty}+\left\|h^{\prime}\right\|_{\infty}+\left\|h^{\prime \prime}\right\|_{\infty}\right)}{k}\right) \mathbb{E}\left|W^{\prime}-W\right|^{3} .
\end{aligned}
$$

Within this framework, we proceed as before: recall that we have defined $W_{n}=\frac{c_{3}}{n^{3 / 2}} \sum_{i, j=1}^{n}\left\langle\sigma_{i}, \sigma_{j}\right\rangle$, and make an exchangeable pair $\left(W_{n}, W_{n}^{\prime}\right)$ by replacing a random spin using the Gibbs sampler. The following lemma gives the bounds needed to apply Theorem 19 in this setting.

Lemma 20. There is a universal constant $C$ such that for $\left(W_{n}, W_{n}^{\prime}\right)$ as constructed above, $k=\frac{2 c_{3}}{3 n^{3 / 2}}$ and $c=\frac{1}{5 c_{3}^{2}}$,

(a) $\mathbb{E}\left[W_{n}^{\prime}-W_{n} \mid \sigma\right]=3 k\left(1-c W_{n}^{2}\right)+R$ and $\mathbb{E}|R| \leq \frac{C \log (n)}{n^{2}} ;$

(b) $\mathbb{E}\left[\left(W_{n}^{\prime}-W_{n}\right)^{2} \mid \sigma\right]=k W_{n}+R^{\prime}$, and $\mathbb{E}\left|R^{\prime}\right| \leq \frac{C \log (n)}{n^{2}}$;

(c) $\mathbb{E}\left|W_{n}^{\prime}-W_{n}\right|^{3} \leq \frac{C \log (n)}{n^{9 / 4}}$.

The proof of Theorem 12 is now immediate. To prove Theorem 13, the same smoothing argument as in the subcritical case can be carried out; again, see Section 3 of [17] for a detailed example.

Proof of Lemma 20. For part (a),

$$
\begin{aligned}
\mathbb{E}\left[W_{n}^{\prime}-W_{n} \mid \sigma\right] & =-\frac{c_{3}}{n^{5 / 2}} \sum_{i=1}^{n}\left[2 \sum_{k \neq i}\left\langle\sigma_{i}, \sigma_{k}\right\rangle-\mathbb{E}\left[2 \sum_{k \neq i}\left\langle\sigma_{i}, \sigma_{k}\right\rangle \mid\left\{\sigma_{j}\right\}_{j \neq i}\right]\right] \\
& =-\frac{2}{n} W_{n}+\frac{2 c_{3}}{n^{3 / 2}}+\frac{2 c_{3}}{n^{5 / 2}} \sum_{i=1}^{n} g\left(\frac{3\left|\sigma^{(i)}\right|}{n}\right)\left|\sigma^{(i)}\right|
\end{aligned}
$$

just as in the supercritical case, again using the notation $g(x)=\operatorname{coth}(x)-\frac{1}{x}$. Now, near zero, $g(x)=\frac{x}{3}-\frac{x^{3}}{45}+O\left(x^{5}\right)$. Using this above,

$$
\sum_{i=1}^{n} g\left(\frac{3\left|\sigma^{(i)}\right|}{n}\right)\left|\sigma^{(i)}\right|=\sum_{i=1}^{n}\left[\frac{\left|\sigma^{(i)}\right|^{2}}{n}-\frac{\left|\sigma^{(i)}\right|^{4}}{5 n^{3}}+O\left(\frac{\left|\sigma^{(i)}\right|^{6}}{n^{5}}\right)\right] .
$$

Note that

$$
\frac{1}{n} \sum_{i=1}^{n}\left|\sigma^{(i)}\right|^{2}=\sum_{j, k}\left\langle\sigma_{j}, \sigma_{k}\right\rangle-\frac{2}{n} \sum_{i, j}\left\langle\sigma_{i}, \sigma_{j}\right\rangle+1=\frac{n^{3 / 2} W_{n}}{c_{3}}-\frac{2 \sqrt{n} W_{n}}{c_{3}}+1 .
$$


Similarly,

$$
-\frac{1}{5 n^{3}} \sum_{i=1}^{n}\left|\sigma^{(i)}\right|^{4}=-\frac{n W_{n}^{2}}{5 c_{3}^{2}}+\frac{4 W_{n}^{2}}{5 c_{3}^{2}}-\frac{4 \sum_{i}\left\langle\sigma_{i}, S_{n}\right\rangle^{2}}{5 n^{3}}-\frac{2 W_{n}}{5 c_{3} \sqrt{n}}+\frac{W_{n}}{5 n^{3 / 2} c_{3}}+\frac{1}{5 n^{2}} .
$$

Using these expressions in (20) yields

$$
\mathbb{E}\left[W_{n}^{\prime}-W_{n} \mid \sigma\right]=\frac{2 c_{3}}{n^{3 / 2}}-\frac{2 W_{n}^{2}}{5 c_{3} n^{3 / 2}}+R
$$

where

$$
R=-\frac{4 W_{n}}{n^{2}}+\frac{8 W_{n}^{2}}{5 c_{3} n^{5 / 2}}+\frac{C W_{n}^{3}}{n^{2} c_{3}^{2}}+\frac{\tilde{R}(\sigma)}{n^{5 / 2}},
$$

$C$ is a universal constant, and furthermore, $\tilde{R}(\sigma) \leq C$ almost surely. Note in particular the cancellation of the $\frac{2}{n} W_{n}$ terms, which is the crucial difference from the subcritical case, and the reason that the limiting distribution of the total spin is not Gaussian for $\beta=3$.

Recall that the LDP for $\mu_{\sigma, n}$ gives us that $\mathbb{E} W_{n}, \mathbb{E} W_{n}^{2}, \mathbb{E} W_{n}^{3} \leq \log (n)$, and so it follows that $\mathbb{E}|R| \leq \frac{C \log (n)}{n^{2}}$. This completes the proof of $(a)$.

For part (b), from the definition as before,

$$
\mathbb{E}\left[\left(W_{n}^{\prime}-W_{n}\right)^{2} \mid \sigma\right]=\frac{c_{3}^{2}}{n^{4}} \sum_{i=1}^{n} \sum_{j, k \neq i} \mathbb{E}\left[\sigma_{j}^{T}\left(\sigma_{i}^{*}-\sigma_{i}\right)\left(\sigma_{i}^{*}-\sigma_{i}\right)^{T} \sigma_{k} \mid \sigma, I=i\right] .
$$

Now, $\mathbb{E}\left[\left(\sigma_{i}^{*}-\sigma_{i}\right)\left(\sigma_{i}^{*}-\sigma_{i}\right)^{T} \mid \sigma, I=i\right]$ was computed exactly in the $\beta<3$ case, and was found to be

$$
\begin{array}{r}
\mathbb{E}\left[\left(\sigma_{i}^{*}-\sigma_{i}\right)\left(\sigma_{i}^{*}-\sigma_{i}\right)^{T} \mid \sigma, I=i\right]=\left\{\left[1-\frac{2 c_{i} \operatorname{coth}\left(c_{i}\right)-2}{c_{i}^{2}}\right] P_{i}+\left[\frac{\operatorname{coth}\left(c_{i}\right)}{c_{i}}-\frac{1}{c_{i}^{2}}\right] P_{i}^{\perp}\right. \\
\left.-\left[\operatorname{coth}\left(c_{i}\right)-\frac{1}{c_{i}}\right]\left(r_{i} \sigma_{i}^{T}+\sigma_{i} r_{i}^{T}\right)+\sigma_{i} \sigma_{i}^{T}\right\},
\end{array}
$$

where $c_{i}=\frac{\beta\left|\sigma^{(i)}\right|}{n}, r_{i}=\frac{\sigma^{(i)}}{\left|\sigma^{(i)}\right|}, P_{i}$ is orthogonal projection onto $r_{i}$, and $P_{i}^{\perp}$ is orthogonal projection onto the orthogonal complement of $r_{i}$.

Recall that $g(x)=\operatorname{coth}(x)-\frac{1}{x} \approx \frac{x}{3}$ for small $x$. Using this above,

$$
\mathbb{E}\left[\left(\sigma_{i}^{*}-\sigma_{i}\right)\left(\sigma_{i}^{*}-\sigma_{i}\right)^{T} \mid \sigma, I=i\right]=\frac{1}{3} I d-\frac{c_{i}}{3}\left(r_{i} \sigma_{i}^{T}+\sigma_{i} r_{i}^{T}\right)+\sigma_{i} \sigma_{i}^{T}+R_{i}^{\prime},
$$

where

$$
R_{i}^{\prime}=\left(\frac{g\left(c_{i}\right)}{c_{i}}-\frac{1}{3}\right) I d-\left(g\left(c_{i}\right)-\frac{c_{i}}{3}\right)\left(r_{i} \sigma_{i}^{T}+\sigma_{i} r_{i}^{T}\right) .
$$

Ignoring the $R_{i}^{\prime}$ for the moment and putting the main term of (22) into (21) yields

$$
\frac{c_{3}^{2}}{n^{4}} \sum_{i} \sum_{j, k \neq i}\left[\frac{1}{3}\left\langle\sigma_{j}, \sigma_{k}\right\rangle-\frac{c_{i}}{3}\left(\sigma_{j}^{T} r_{i} \sigma_{i}^{T} \sigma_{k}+\sigma_{j}^{T} \sigma_{i} r_{i}^{T} \sigma_{k}\right)+\sigma_{j}^{T} \sigma_{i} \sigma_{i}^{T} \sigma_{k}\right]
$$


The first term is

$$
\frac{c_{3}^{2}}{3 n^{4}} \sum_{i} \sum_{j, k \neq i}\left\langle\sigma_{j}, \sigma_{k}\right\rangle=\frac{c_{3}^{2}}{3 n^{4}} \sum_{i=1}^{n}\left|\sigma^{(i)}\right|^{2}
$$

The first half of the second term is

$$
-\frac{c_{3}^{2}}{3 n^{4}} \sum_{i} \sum_{j, k \neq i} c_{i} \sigma_{j}^{T} r_{i} \sigma_{i}^{T} \sigma_{k}=-\frac{c_{3}^{2}}{3 n^{4}} \sum_{i} c_{i}\left|\sigma^{(i)}\right|\left\langle\sigma^{(i)}, \sigma_{i}\right\rangle=-\frac{c_{3}^{2}}{n^{5}} \sum_{i}\left|\sigma^{(i)}\right|^{2}\left\langle\sigma^{(i)}, \sigma_{i}\right\rangle,
$$

and the second half is the same. Finally,

$$
\frac{c_{3}^{2}}{n^{4}} \sum_{i} \sum_{j, k \neq i} \sigma_{j}^{T} \sigma_{i} \sigma_{i}^{T} \sigma_{k}=\frac{c_{3}^{2}}{n^{4}} \sum_{i}\left\langle\sigma_{i}, \sigma^{(i)}\right\rangle^{2}
$$

all together,

$$
\begin{aligned}
\mathbb{E}\left[\left(W_{n}^{\prime}-W_{n}\right)^{2} \mid \sigma\right]=\frac{c_{3}^{2}}{n^{4}} \sum_{i} & {\left[\frac{1}{3}\left|\sigma^{(i)}\right|^{2}-\frac{2}{n}\left|\sigma^{(i)}\right|^{2}\left\langle\sigma_{i}, \sigma^{(i)}\right\rangle+\left\langle\sigma_{i}, \sigma^{(i)}\right\rangle^{2}\right] } \\
& \quad+\frac{c_{3}^{2}}{n^{4}} \sum_{i} \sum_{j, k \neq i} \sigma_{j}^{T} R_{i}^{\prime} \sigma_{k} \\
= & \frac{c_{3}^{2}}{n^{4}} \sum_{i}\left[\frac{2}{3}\left|\sigma^{(i)}\right|^{2}-\frac{2}{n}\left|\sigma^{(i)}\right|^{2}\left\langle\sigma_{i}, \sigma^{(i)}\right\rangle\right]+\frac{c_{3}^{2}}{n^{4}} \sum_{i} \sum_{j, k \neq i} \sigma_{j}^{T} R_{i}^{\prime} \sigma_{k} \\
= & \frac{2 c_{3}^{2}}{3 n^{3}}\left(\left|S_{n}\right|^{2}-\frac{\left|S_{n}\right|^{2}}{n}+1\right)-\frac{2 c_{3}^{2}}{n^{5}} \sum_{i}\left|\sigma^{(i)}\right|^{2}\left\langle\sigma_{i}, \sigma^{(i)}\right\rangle+\frac{c_{3}^{2}}{n^{4}} \sum_{i} \sum_{j, k \neq i} \sigma_{j}^{T} R_{i}^{\prime} \sigma_{k},
\end{aligned}
$$

where the computation for $\mathbb{E}\left[\left\langle\sigma_{i}, \sigma^{(i)}\right\rangle^{2}\right]$ from the supercritical case has been used. Recall that the main term should be $k W_{n}=\frac{2 c_{3} W_{n}}{3 n^{3 / 2}}$ and indeed it is. It is a routine collection of arguments very similar to those in the previous sections to show that the remaining terms are bounded in expectation by $\frac{C \log (n)}{n^{2}}$.

Finally, part $(c)$ is straightforward as usual:

$$
\begin{aligned}
\mathbb{E}\left|W_{n}^{\prime}-W_{n}\right|^{3}=\frac{8 c_{3}^{3}}{n^{9 / 2}} \mathbb{E}\left|\sum_{j \neq I}\left\langle\sigma_{I}^{*}-\sigma_{I}, \sigma_{j}\right\rangle\right|^{3} & =\frac{8 c_{3}^{3}}{n^{9 / 2}} \mathbb{E}\left|\left\langle\sigma_{I}^{*}-\sigma_{I}, S_{n}-\sigma_{I}\right\rangle\right|^{3} \\
& \leq \frac{8 c_{3}^{3}}{n^{9 / 2}}\left[8 \mathbb{E}\left|S_{n}\right|^{3}+8\right] \leq \frac{C \log (n)}{n^{9 / 4}} .
\end{aligned}
$$

\section{Appendix}

\subsection{Calculus of $\Phi_{\beta}$}

Recall that the free energy is obtained by minimizing the function

$$
\Phi_{\beta}(x):=\log \left(\frac{x}{\sinh (x)}\right)+x \operatorname{coth}(x)-1-\frac{\beta}{2}\left(\operatorname{coth}(x)-\frac{1}{x}\right)^{2} .
$$


In the following lemmas, we explicitly identify the minima for all $\beta>0$, and obtain an estimate used in the proof of Lemma 14.

Lemma 21. If $\beta \leq 3$, then

$$
\inf _{x \geq 0}\left\{\log \left(\frac{x}{\sinh (x)}\right)+x\left(\operatorname{coth}(x)-\frac{1}{x}\right)-\frac{\beta}{2}\left(\operatorname{coth}(x)-\frac{1}{x}\right)^{2}\right\}=0,
$$

achieved only at $x=0$.

Proof. We show first that the expression to be minimized is increasing. Differentiating the expression in question yields

$$
\begin{aligned}
& {\left[\frac{\sinh (x)}{x}\right]\left[\frac{\sinh (x)-x \cosh (x)}{\sinh ^{2}(x)}\right]+\left[\operatorname{coth}(x)-\frac{1}{x}\right]+x\left[\frac{1}{x^{2}}-\frac{1}{\sinh ^{2}(x)}\right]} \\
& -\beta\left[\operatorname{coth}(x)-\frac{1}{x}\right]\left[\frac{1}{x^{2}}-\frac{1}{\sinh ^{2}(x)}\right] \\
& =\left[\frac{1}{x^{2}}-\frac{1}{\sinh ^{2}(x)}\right]\left[x-\beta\left(\operatorname{coth}(x)-\frac{1}{x}\right)\right] .
\end{aligned}
$$

Expanding $\sinh (x)$ in a Taylor series,

$$
\sinh ^{2}(x)=\left(x+\sum_{n=1}^{\infty} \frac{x^{2 n+1}}{(2 n+1) !}\right)^{2}>x^{2} .
$$

The problem is therefore reduced to showing that

$$
x-\beta\left(\operatorname{coth}(x)-\frac{1}{x}\right)>0,
$$

or alternatively,

$$
\beta<\frac{x^{2}}{x \operatorname{coth}(x)-1}
$$

Clearly showing this for $\beta=3$ suffices to prove the lemma. Rearranging yet again, this is equivalent to showing that

$$
\operatorname{coth}(x)-\frac{1}{x}<\frac{x}{3}
$$

Expanding $\operatorname{coth}(x)$ in terms of $e^{2 x}$ and rearranging terms, this is furthermore equivalent to showing that

$$
\left(1-x+\frac{x^{2}}{3}\right) e^{2 x}>1+x+\frac{x^{2}}{3} .
$$

Expanding $e^{2 x}$ in a Taylor series, the left-hand side of the inequality above is given by

$$
\begin{aligned}
1+x+\frac{x^{2}}{3}+\sum_{n=3}^{\infty} x^{n} & {\left[\frac{2^{n}}{n !}-\frac{2^{n-1}}{(n-1) !}+\frac{2^{n-2}}{3(n-2) !}\right] } \\
& =1+x+\frac{x^{2}}{3}+\sum_{n=3}^{\infty} \frac{2^{n-2} x^{n}}{3 n !}\left[\left(n-\frac{7}{2}\right)^{2}-\frac{1}{4}\right] .
\end{aligned}
$$


It is easy to see that the $n=3$ and $n=4$ terms in the power series above are zero and that the rest are all positive, thus completing the proof that the expression to be minimized is increasing. Moreover, recall that $\lim _{x \rightarrow 0} \operatorname{coth}(x)-\frac{1}{x}=0$ and $\lim _{x \rightarrow 0} \frac{x}{\sinh (x)}=1$, so

$$
\lim _{x \rightarrow 0}\left\{\log \left(\frac{x}{\sinh (x)}\right)+x\left(\operatorname{coth}(x)-\frac{1}{x}\right)-\frac{\beta}{2}\left(\operatorname{coth}(x)-\frac{1}{x}\right)^{2}\right\}=0 .
$$

Lemma 22. For $\beta>3$, there is a unique value of $x \in(0, \infty)$ which minimizes

$$
\log \left(\frac{x}{\sinh (x)}\right)+x\left(\operatorname{coth}(x)-\frac{1}{x}\right)-\frac{\beta}{2}\left(\operatorname{coth}(x)-\frac{1}{x}\right)^{2}
$$

over $[0, \infty)$.

Proof. From the previous proof, we have that the derivative of the expression to be minimized is

$$
\left[\frac{1}{x^{2}}-\frac{1}{\sinh ^{2}(x)}\right]\left[x-\beta\left(\operatorname{coth}(x)-\frac{1}{x}\right)\right] \sim \frac{(3-\beta) x}{9},
$$

for $x$ near zero. For $\beta>3$, it follows that $x=0$ is a local maximum of the expression on $[0, \infty)$. As $x$ tends to infinity, the expression to be minimized is asymptotic to $\log (x)$, and there is therefore at least one interior minimum. Since $\frac{1}{x^{2}}-\frac{1}{\sinh ^{2}(x)}>0$, it must be the case that at this interior minimum,

$$
x-\beta\left(\operatorname{coth}(x)-\frac{1}{x}\right)=0
$$

that is,

$$
\beta=\frac{x}{\operatorname{coth}(x)-\frac{1}{x}}
$$

In fact, the function $g(x)=\frac{x}{\operatorname{coth}(x)-\frac{1}{x}}$ is strictly increasing on $(0, \infty)$, and this equation thus uniquely determines $x$ in terms of $\beta$. First observe that

$$
g^{\prime}(x)=\frac{\operatorname{coth}(x)-\frac{2}{x}+\frac{x}{\sinh ^{2}(x)}}{\left(\operatorname{coth}(x)-\frac{1}{x}\right)^{2}}
$$

and it thus suffices to show that

$$
\operatorname{coth}(x)-\frac{2}{x}+\frac{x}{\sinh ^{2}(x)}>0
$$

for $x>0$; multiplying through by $x \sinh ^{2}(x)$, one could equivalently show that

$$
x \sinh (x) \cosh (x)+x^{2}-2 \sinh ^{2}(x)>0 .
$$


Using the identities $\sinh (x) \cosh (x)=\frac{\sinh (2 x)}{2}$ and $\sinh ^{2}(x)=\frac{\cosh (2 x)-1}{2}$, this is equivalent to showing that

$$
\frac{x}{2} \sinh (2 x)+x^{2}-\cosh (2 x)+1>0 .
$$

Expanding the left-hand side in Taylor series yields

$$
\sum_{n=2}^{\infty} x^{2 n}\left[\frac{2^{2 n-1}}{2(2 n-1) !}-\frac{2^{2 n}}{(2 n) !}\right]=\sum_{n=3}^{\infty} \frac{x^{2 n} 2^{2 n-2}}{(2 n) !}[2 n-4]
$$

all of whose terms are indeed positive.

Lemma 23. Let $k_{2}$ denote the unique value of $x \in(0, \infty)$ with

$$
x-\beta\left(\operatorname{coth}(x)-\frac{1}{x}\right)=0 .
$$

Then

$$
\beta\left(\frac{1}{k_{2}^{2}}-\frac{1}{\sinh ^{2}\left(k_{2}\right)}\right)<1
$$

In particular, if

$$
\Phi_{\beta}(x):=\log \left(\frac{x}{\sinh (x)}\right)+x\left(\operatorname{coth}(x)-\frac{1}{x}\right)-\frac{\beta}{2}\left(\operatorname{coth}(x)-\frac{1}{x}\right)^{2}
$$

then $\Phi_{\beta}^{\prime}\left(k_{2}\right)=0$ and $\Phi_{\beta}^{\prime \prime}\left(k_{2}\right)>0$.

Proof. Let

$$
f(x):=x-\beta\left(\operatorname{coth}(x)-\frac{1}{x}\right)
$$

so that $f\left(k_{2}\right)=0$; as was shown in the previous proof, this uniquely defines $k_{2}$ in terms of $\beta$. Moreover, it was also shown that $\lim _{x \rightarrow 0} f(x)=0, \lim _{x \rightarrow \infty} f(x)=\infty$, and $\lim _{x \rightarrow 0} f^{\prime}(x)<0$. That is, $f$ is initially decreasing from 0 , and then becomes increasing eventually, crossing the $x$-axis exactly once in $(0, \infty)$. It must therefore be that there is an $x<k_{2}$ such that $f^{\prime}(x)=0$. Now,

$$
f^{\prime}(x)=1-\beta\left(\frac{1}{x^{2}}-\frac{1}{\sinh ^{2}(x)}\right) .
$$

In fact, $g(x):=\frac{1}{x^{2}}-\frac{1}{\sinh ^{2}(x)}$ is decreasing on $(0, \infty)$ : observe first that

$$
g^{\prime}(x)=2 \operatorname{coth}(x) \operatorname{csch}^{2}(x)-\frac{2}{x^{3}},
$$

and so the claim is true if $\operatorname{coth}(x) \operatorname{csch}^{2}(x)<x^{-3}$. By the definitions of the hyperbolic trigonometric functions, this is equivalent to

$$
4 x^{3}\left(e^{4 x}+e^{2 x}\right)<\left(e^{2 x}-1\right)^{3} .
$$


Expanding in power series, this is equivalent to

$$
\sum_{n=3}^{\infty} \frac{4\left(4^{n-3}+2^{n-3}\right)}{(n-3) !} x^{n}<\left(\sum_{n=1}^{\infty} \frac{(2 x)^{n}}{n !}\right)^{3}=\sum_{n=3}^{\infty}\left(\sum_{\substack{i, j, k \geq 1 \\ i+j+k=n}} \frac{2^{n}}{i ! j ! k !}\right) x^{n} .
$$

Letting $\alpha:=i-1, \beta:=j-1$, and $\gamma=k-1$, the coefficient of $x^{n}$ on the right-hand side is

$$
\frac{2^{n}}{(n-3) !} \sum_{\substack{\alpha, \beta, \gamma \geq 0 \\
\alpha+\beta+\gamma=n-3}}\left(\begin{array}{c}
n-3 \\
\alpha, \beta, \gamma
\end{array}\right)=\frac{2^{n}}{(n-3) !} 3^{n-3}
$$

It is now easy to see that the coefficient of $x^{n}$ on the right-hand side is smaller than the one on the left for each $n \geq 3$, and so it is in fact true that $g(x):=\frac{1}{x^{2}}-\frac{1}{\sinh ^{2}(x)}$ is decreasing on $(0, \infty)$. It follows that $f^{\prime}(x)$ is increasing on $(0, \infty)$, and so the previously identified $x<k_{2}$ such that $f^{\prime}(x)=0$ is in fact the only zero of $f^{\prime}$, and $f^{\prime}\left(k_{2}\right)>0$.

Now, recall from the proof of the previous lemma that

$$
\Phi_{\beta}^{\prime}(x)=\left[\frac{1}{x^{2}}-\frac{1}{\sinh ^{2}(x)}\right]\left[x-\beta\left(\operatorname{coth}(x)-\frac{1}{x}\right)\right] .
$$

The value $k_{2}$ was in fact determined by the fact that $\Phi_{\beta}^{\prime}\left(k_{2}\right)=0$. Moreover,

$$
\begin{aligned}
\Phi_{\beta}^{\prime \prime}(x)=\left[\frac{1}{x^{2}}-\right. & \left.\frac{1}{\sinh ^{2}(x)}\right]\left[1-\beta\left(\frac{1}{x^{2}}-\frac{1}{\sinh ^{2}(x)}\right)\right] \\
& +\left[2 \operatorname{coth}(x) \operatorname{csch}^{2}(x)-\frac{2}{x^{3}}\right]\left[x-\beta\left(\operatorname{coth}(x)-\frac{1}{x}\right)\right],
\end{aligned}
$$

and so

$$
\Phi_{\beta}^{\prime \prime}\left(k_{2}\right)=\left[\frac{1}{k_{2}^{2}}-\frac{1}{\sinh ^{2}\left(k_{2}\right)}\right]\left[1-\beta\left(\frac{1}{k_{2}^{2}}-\frac{1}{\sinh ^{2}\left(k_{2}\right)}\right)\right]>0
$$

\subsection{Proof of Theorem 19}

This section is devoted to the proof of the abstract approximation theorem used in Section 6. For convenience, we recall the statement.

Theorem 19. Let $\left(W, W^{\prime}\right)$ be an exchangeable pair of positive random variables. Suppose there exists a $\sigma$-field $\mathcal{F} \supseteq \sigma(W)$ and $k>0$ deterministic such that

$$
\mathbb{E}\left[W^{\prime}-W \mid \mathcal{F}\right]=3 k\left(1-c W^{2}\right)+E
$$

and

$$
\mathbb{E}\left[\left(W^{\prime}-W\right)^{2} \mid \mathcal{F}\right]=k W+E^{\prime}
$$


Let $X$ have density

$$
p(t)= \begin{cases}\frac{1}{z} x^{5} e^{-\frac{c t^{2}}{2}} & t \geq 0 \\ 0 & t<0 .\end{cases}
$$

Then there are constants $C_{1}, C_{2}, C_{3}$ depending only on $c$ such that for all $h \in C^{2}(\mathbb{R})$,

$$
\begin{aligned}
|\mathbb{E} h(W)-\mathbb{E} h(X)| \leq & \frac{C_{1}\|h\|_{\infty}}{k} \mathbb{E}|E|+\left(\frac{C_{2}\left(\|h\|_{\infty}+\left\|h^{\prime}\right\|_{\infty}\right)}{k}\right) \mathbb{E}\left|E^{\prime}\right| \\
& +\left(\frac{C_{3}\left(\|h\|_{\infty}+\left\|h^{\prime}\right\|_{\infty}+\left\|h^{\prime \prime}\right\|_{\infty}\right)}{k}\right) \mathbb{E}\left|W^{\prime}-W\right|^{3} .
\end{aligned}
$$

In any version of Stein's method, a crucial component is the characterization of the distribution of interest by a linear operator. The following lemma identifies the characterizing operator for the random variable $X$ defined above.

Lemma 24. Let $Y$ be a positive random variable. Then $Y$ has density

$$
p(t)= \begin{cases}\frac{1}{z} t^{5} e^{-3 c t^{2}} & t \geq 0 \\ 0 & t<0\end{cases}
$$

if and only if

$$
\mathbb{E}\left[Y f^{\prime}(Y)+6\left(1-c Y^{2}\right) f(Y)\right]=0
$$

for all $f \in C^{1}((0, \infty))$ such that $\int_{0}^{\infty} f(t) t^{5} e^{-3 c t^{2}} d t<\infty$. That is, the characterizing operator $T_{p}$ for the distribution with density $p$ is defined by

$$
\left[T_{p} f\right](x)=x f^{\prime}(x)+6\left(1-c x^{2}\right) f(x)
$$

Not only is the random variable $X$ characterized by the operator above, but this operator is invertible on $\{h: \mathbb{E} h(X)=0\}$, and the inverse has the following important boundedness properties.

Lemma 25. Let $h: \mathbb{R} \rightarrow \mathbb{R}$ be given. Suppose that

$$
f=f_{h}:=\frac{1}{t p(t)} \int_{0}^{t}[h(s)-\mathbb{E} h(X)] p(s) d s,
$$

with $p$ as above. Then $\left[T_{p} f_{h}\right](x)=h(x)-\mathbb{E} h(X)$ and

(a) $\left\|f_{h}\right\|_{\infty} \leq 5\|h\|_{\infty}$.

(b) $\left\|f_{h}^{\prime}\right\|_{\infty} \leq 42 \sqrt{c}\|h\|_{\infty}+3\left\|h^{\prime}\right\|_{\infty}$.

(c) $\left\|f_{h}^{\prime \prime}\right\|_{\infty} \leq C_{1}\|h\|_{\infty}+C_{2}\left\|h^{\prime}\right\|_{\infty}+C_{3}\left\|h^{\prime \prime}\right\|_{\infty}$, where $C_{1}, C_{2}, C_{3}$ are constants depending only on $c$.

With these two lemmas, the proof of Theorem 19 is relatively straightforward. 
Proof of Theorem [19. Given $h$, let $f$ be the solution to the Stein equation described above. Then by exchangeability and the conditions on $\left(W, W^{\prime}\right)$,

$$
\begin{aligned}
0 & =\mathbb{E}\left[\left(W^{\prime}-W\right)\left(f\left(W^{\prime}\right)+f(W)\right)\right] \\
& =\mathbb{E}\left[\left(W^{\prime}-W\right)\left(f\left(W^{\prime}\right)-f(W)\right)+2\left(W^{\prime}-W\right) f(W)\right] \\
& =\mathbb{E}\left[\left(W^{\prime}-W\right)^{2} f^{\prime}(W)+E^{\prime \prime}+6 k\left(1-c W^{2}\right) f(W)+2 E f(W)\right] \\
& =\mathbb{E}\left[k W f^{\prime}(W)+E^{\prime} f^{\prime}(W)+E^{\prime \prime}+6 k\left(1-c W^{2}\right) f(W)+2 E f(W)\right] .
\end{aligned}
$$

Then

$$
\mathbb{E}\left[W f^{\prime}(W)+6\left(1-c W^{2}\right) f(W)\right]=-\frac{1}{k} \mathbb{E}\left[E^{\prime} f^{\prime}(W)+2 E f(W)+E^{\prime \prime}\right]
$$

and

$$
\left|E^{\prime \prime}\right| \leq \frac{\left\|f^{\prime \prime}\right\|_{\infty}}{2}\left|\left(W^{\prime}-W\right)\right|^{3}
$$

The result is thus immediate from Lemma 25.

We conclude by giving the proofs of the key lemmas.

Proof of Lemma 24. If $Y$ has the density above, then the fact that $Y$ satisfies (23) is just integration by parts.

For the reverse implication, one must solve the so-called Stein equation; i.e., given $h$ : $(0, \infty) \rightarrow \mathbb{R}$, find $f=f_{h}$ such that

$$
t f^{\prime}(t)+6\left(1-c t^{2}\right) f(t)=h(t)-\mathbb{E} h(X),
$$

where $X$ has the density above. The solution $f$ is given by

$$
\begin{aligned}
f(t) & =\frac{1}{t p(t)} \int_{0}^{t}[h(s)-\mathbb{E} h(X)] p(s) d s \\
& =-\frac{1}{t p(t)} \int_{t}^{\infty}[h(s)-\mathbb{E} h(X)] p(s) d s,
\end{aligned}
$$

where $p(s):=\frac{s^{5}}{z} e^{-3 c s^{2}}$. Observe that

$$
\frac{d}{d t}[t f(t) p(t)]=\left[f(t)+t f^{\prime}(t)\right] p(t)+t f(t) p^{\prime}(t)=[h(t)-\mathbb{E} h(X)] p(t),
$$

so that

$$
h(t)-\mathbb{E} h(X)=f(t)+t f^{\prime}(t)+\frac{t f(t) p^{\prime}(t)}{p(t)}=6\left(1-c t^{2}\right) f(t)+t f^{\prime}(t) .
$$

Here we have made use of the fact, frequently used below, that

$$
\frac{t p^{\prime}(t)}{p(t)}=5-6 c t^{2} \text {. }
$$

It is shown in the next lemma that $f$ and $f^{\prime}$ are both bounded, and so if $Y$ satisfies (23), then if $h$ is given and $f$ solves the Stein equation,

$$
\mathbb{E} h(Y)-\mathbb{E} h(X)=\mathbb{E}\left[Y f^{\prime}(Y)+6\left(1-c Y^{2}\right) f(Y)\right]=0,
$$

and so $Y \stackrel{d}{=} X$. 
Proof of Lemma 25. (a) By the first expression for $f_{h}$, if $t \leq t_{0}:=\sqrt{\frac{5}{6 c}}$, then

$$
f(t) \leq \frac{2\|h\|_{\infty}}{t p(t)}\left(\int_{0}^{t} p(s) d s\right) \leq \frac{\|h\|_{\infty} t^{5}}{3 z p(t)} \leq \frac{e^{5 / 2}\|h\|_{\infty}}{3} \leq 5\|h\|_{\infty} .
$$

By the second expression for $f_{h}$,

$$
|f(t)| \leq \frac{2\|h\|_{\infty} \mathbb{P}[X \geq t]}{t p(t)}
$$

It is easy to show directly that $\mathbb{P}[X \geq t] \leq \frac{p(t)}{6 c t}\left(1+\frac{2}{3 c t^{2}}+\frac{2}{9 c^{2} t^{4}}\right)$, and so

$$
|f(t)| \leq \frac{2\|h\|_{\infty}}{6 c t^{2}}\left(1+\frac{2}{3 c t^{2}}+\frac{2}{9 c^{2} t^{4}}\right) \leq \frac{84\|h\|_{\infty}}{125}
$$

for $t \geq t_{o}$. This completes the proof.

(b) Recall that, because $f$ solves the Stein equation,

$$
t f^{\prime}(t)=6 f(t)\left(c t^{2}-1\right)+h(t)-\mathbb{E} h(X) .
$$

For $t \leq t_{o}$, observe that

$$
\begin{aligned}
h(t)-\mathbb{E} h(X)-6 f(t) & =h(t)-\mathbb{E} h(X)-\frac{6}{t p(t)} \int_{0}^{t}[h(s)-\mathbb{E} h(X)] p(s) d s \\
& =\frac{6}{t p(t)} \int_{0}^{t}\left([h(t)-\mathbb{E} h(X)] \frac{s^{5} p(t)}{t^{5}}-[h(s)-\mathbb{E} h(X)] p(s)\right) d s
\end{aligned}
$$

Now,

$$
\begin{gathered}
\left|\frac{1}{t p(t)} \int_{0}^{t}[h(t)-\mathbb{E} h(X)]\left(\frac{s^{5} p(t)}{t^{5}}-p(s)\right) d s\right| \\
\leq \frac{2\|h\|_{\infty}}{t p(t)} \int_{0}^{t}\left|1-\frac{s^{5} p(t)}{t^{5} p(s)}\right| p(s) d s=\frac{2\|h\|_{\infty}}{t p(t)} \int_{0}^{t}\left|1-e^{3 c\left(s^{2}-t^{2}\right)}\right| p(s) d s \\
\leq 2\|h\|_{\infty} c t^{2}
\end{gathered}
$$

making use of the fact that $p(s) \leq p(t)$ in this range. Also,

$$
\begin{array}{r}
\left|\frac{1}{t p(t)} \int_{0}^{t}([h(t)-\mathbb{E} h(X)]-[h(s)-\mathbb{E} h(X)]) p(s) d s\right| \\
\leq \frac{\left\|h^{\prime}\right\|_{\infty}}{t p(t)} \int_{0}^{t}(t-s) p(s) d s \leq \frac{\left\|h^{\prime}\right\|_{\infty} t}{2},
\end{array}
$$

so that for $t \leq t_{o}$,

$$
\frac{1}{t}|h(t)-\mathbb{E} h(X)-6 f(t)| \leq 12\|h\|_{\infty} c t_{o}+3\left\|h^{\prime}\right\|_{\infty},
$$


and thus

$$
\begin{aligned}
\left|f^{\prime}(t)\right| & \leq 6 c t_{o}\|f\|_{\infty}+12\|h\| c t_{o}+3\left\|h^{\prime}\right\|_{\infty} \\
& \leq 42 c t_{o}\|h\|_{\infty}+3\left\|h^{\prime}\right\|_{\infty} .
\end{aligned}
$$

If $t \geq t_{o}$, then

$$
\left|f^{\prime}(t)\right| \leq 6 c t|f(t)|+\frac{6\|f\|_{\infty}+2\|h\|_{\infty}}{t_{o}} .
$$

By the second expression for $f$,

$$
c t|f(t)| \leq \frac{2 c\|h\|_{\infty} \mathbb{P}[X \geq t]}{p(t)} \leq \frac{\|h\|_{\infty}}{3 t}\left(1+\frac{2}{3 c t^{2}}+\frac{2}{9 c^{2} t^{4}}\right),
$$

making use again of the estimate $\mathbb{P}[X \geq t] \leq \frac{p(t)}{6 c t}\left(1+\frac{2}{3 c t^{2}}+\frac{2}{9 c^{2} t^{4}}\right)$. Taking $t_{o}=\sqrt{\frac{5}{6 c}}$ as before and making some trivial simplifying estimates completes the proof of this part.

(c) Differentiating both sides of the Stein equation gives that

$$
t f^{\prime \prime}(t)=12 c t f(t)+\left(6 c t^{2}-7\right) f^{\prime}(t)+h^{\prime}(t) .
$$

If $t \leq t_{o}$, then $|12 c f(t)| \leq 5 c\|h\|_{\infty}$ and by the Stein equation, $\left|6 c t f^{\prime}(t)\right| \leq 36 c\|f\|_{\infty}+$ $12 c\|h\|_{\infty} \leq 192 c\|h\|_{\infty}$. Also by the Stein equation,

$$
f^{\prime}(t)=6 f(t)\left(c t-\frac{1}{t}\right)+\frac{h(t)-\mathbb{E} h(X)}{t} .
$$

The first term can be absorbed into the existing bound, so that

$$
\left|f^{\prime \prime}(t)\right| \leq C\|h\|_{\infty}+\frac{1}{t}\left|h^{\prime}(t)+\frac{42 f(t)-7[h(t)-\mathbb{E} h(X)]}{t}\right| .
$$

(From now on we will not bother to keep track of specific constants and their dependence on c.) Now,

$$
\begin{aligned}
h^{\prime}(t) & -\frac{7[h(t)-\mathbb{E} h(X)-6 f(t)]}{t} \\
& =h^{\prime}(t)-\frac{42}{t^{2} p(t)} \int_{0}^{t}\left([h(t)-\mathbb{E} h(X)] \frac{s^{5} p(t)}{t^{5}}-[h(s)-\mathbb{E} h(X)] p(s)\right) d s \\
& =-\frac{42}{t^{2} p(t)} \int_{0}^{t}\left([h(t)-\mathbb{E} h(X)] \frac{s^{5} p(t)}{t^{5}}-[h(s)-\mathbb{E} h(X)] p(s)-\frac{(t-s) s^{5} h^{\prime}(t) p(t)}{t^{5}}\right) d s \\
& =-\frac{42}{t^{2} p(t)} \int_{0}^{t}\left([h(t)-\mathbb{E} h(X)] e^{-3 c t^{2}}-[h(s)-\mathbb{E} h(X)] e^{-3 c s^{2}}-(t-s) h^{\prime}(t) e^{-3 c t^{2}}\right) \frac{s^{5}}{z} d s .
\end{aligned}
$$

Let $H(t):=[h(t)-\mathbb{E} h(X)] e^{-3 c t^{2}}$. Then

$$
H^{\prime}(t)=\left[h^{\prime}(t)-6 c t[h(t)-\mathbb{E} h(X)]\right] e^{-3 c t^{2}},
$$


and so the equation above becomes

$$
\begin{aligned}
h^{\prime}(t) & -\frac{7[h(t)-\mathbb{E} h(X)-6 f(t)]}{t} \\
& =\frac{42}{t^{2} p(t)} \int_{0}^{t}\left(H(s)-H(t)-(s-t) H^{\prime}(t)+6 c t(t-s)[h(t)-\mathbb{E} h(X)] e^{-3 c t^{2}}\right) \frac{s^{5}}{z} d s .
\end{aligned}
$$

It follows that

$$
\left|h^{\prime}(t)-\frac{7[h(t)-\mathbb{E} h(X)-6 f(t)]}{t}\right| \leq \frac{42}{t^{2} p(t)}\left[\left(\sup _{s \in(0, t)}\left|H^{\prime \prime}(s)\right|\right)\left(\frac{t^{8}}{12 z}\right)+\frac{c t^{8}}{z}[h(t)-\mathbb{E} h(X)] e^{-3 c t^{2}}\right] .
$$

Now,

$$
H^{\prime \prime}(s)=\left[h^{\prime \prime}(s)-12 c s h^{\prime}(s)-6 c\left(6 c s^{2}-1\right)[h(s)-\mathbb{E} h(X)]\right] e^{-3 c s^{2}},
$$

SO

$$
\sup _{s \in(0, t)}\left|H^{\prime \prime}(s)\right| \leq\left\|h^{\prime \prime}\right\|_{\infty}+12 c t_{o}\left\|h^{\prime}\right\|_{\infty}+24 c\|h\|_{\infty} .
$$

All together, this gives that for $t \leq t_{o}$,

$$
\left|f^{\prime \prime}(t)\right| \leq C_{1}\|h\|_{\infty}+C_{2}\left\|h^{\prime}\right\|_{\infty}+\left\|h^{\prime \prime}\right\|_{\infty}
$$

where $C_{1}, C_{2}, C_{3}$ are constants depending only on $c$.

For $t>t_{o}$, it follows from (24) and estimates already carried out that

$$
\begin{aligned}
\left|f^{\prime \prime}(t)\right| & \leq 12 c\|f\|_{\infty}+\left(6 c t+\frac{7}{t_{o}}\right)\left|f^{\prime}(t)\right|+\frac{\left\|h^{\prime}\right\|_{\infty}}{t_{o}} \\
& \leq C_{4}\|h\|_{\infty}+C_{5}\left\|h^{\prime}\right\|_{\infty}+c t\left|f^{\prime}(t)\right| .
\end{aligned}
$$

By the Stein equation,

$$
c t f^{\prime}(t)=6 c f(t)\left(c t^{2}-1\right)+c h(t)-c \mathbb{E} h(X),
$$

and

$$
\left|c^{2} t^{2} f(t)\right| \leq \frac{2 c^{2} t\|h\|_{\infty} \mathbb{P}[X \geq t]}{p(t)} \leq \frac{c\|h\|_{\infty}}{3}\left(1+\frac{2}{3 c t^{2}}+\frac{2}{9 c^{2} t^{4}}\right)
$$

and so finally

$$
\left|f^{\prime \prime}(t)\right| \leq C_{6}\|h\|_{\infty}+C_{4}\left\|h^{\prime}\right\|_{\infty}
$$

\section{Acknowledgments}

The authors thank Richard Ellis, Giovanni Gallavotti, and Enzo Marinari for helpful discussions. 


\section{References}

[1] Barbour, Andrew; Chen, Louis. An Introduction to Stein's Method. Lecture Notes Series, Institute for Mathematical Sciences, National University of Singapore, vol. 4 (2005).

[2] Biskup, Marek; Chayes, Lincoln. Rigorous analysis of discontinuous phase transitions via mean-field bounds. Comm. Math. Phys. 238 (2003), no. 1-2, 53-93.

[3] Costeniuc, Marius; Ellis, Richard S.; Touchette, Hugo. Complete analysis of phase transitions and ensemble equivalence for the Curie-Weiss-Potts model. J. Math. Phys. 46 (2005), no. 6, 063301, 25 pp.

[4] Dyson, Freeman J.; Lieb, Elliott H.; Simon, Barry. Phase transitions in quantum spin systems with isotropic and nonisotropic interactions. J. Stat. Phys. 18 (1978), no. 4, pp.335-383.

[5] Chatterjee, Sourav; Shao, Qi-Man. Nonnormal approximation by Stein's method of exchangeable pairs with application to the Curie-Weiss model. Ann. Appl. Probab. 21 (2011), no. 2, 464-483.

[6] Cover, Thomas M. and Thomas, Joy A. Elements of information theory. WileyInterscience [John Wiley \& Sons], Hoboken, NJ, second edition, 2006.

[7] Dembo, Amir; Zeitouni, Ofer. Large Deviations: Techniques and Applications, 2e. Springer, 1998.

[8] Dobrushin, R. L.; Shlosman, S. B. Absence of breakdown of continuous symmetry in two-dimensional models of statistical physics. Comm. Math. Phys. 42 (1975), 31-40.

[9] Eichelsbacher, Peter; Martschink, Bastian. On rates of convergence in the CurieWeiss-Potts model with an external field. arXiv:1011.0319v1.

[10] Ellis, Richard S.; Haven, Kyle; Turkington, Bruce. Large deviation principles and complete equivalence and nonequivalence results for pure and mixed ensembles. J. Statist. Phys. 101 (2000), no. 5-6, 999-1064.

[11] Ellis, Richard S.; Newman, Charles M. Limit theorems for sums of dependent random variables occurring in statistical mechanics. Z. Wahrsch. Verw. Gebiete 44 (1978), no. $2,117-139$.

[12] Ellis, Richard S.; Newman, Charles M.; Rosen, Jay S. Limit theorems for sums of dependent random variables occurring in statistical mechanics. II. Conditioning, multiple phases, and metastability. Z. Wahrsch. Verw. Gebiete 51 (1980), no. 2.

[13] Fröhlich, J.; Simon, B.; Spencer, Thomas Infrared bounds, phase transitions and continuous symmetry breaking. Comm. Math. Phys. 50 (1976), no. 1, 79-95.

[14] Kesten, H.; Schonmann, R. H. Behavior in large dimensions of the Potts and Heisenberg models. Rev. Math. Phys. 1 (1989), no. 2-3, 147-182. 
[15] Malyshev, V. A. Phase transitions in classical Heisenberg ferromagnets with arbitrary parameter of anisotropy. Comm. Math. Phys. 40 (1975), 75-82.

[16] Meckes, E. On Stein's method for multivariate normal approximation. In High Dimensional Probability V: The Luminy Volume (2009).

[17] Meckes, M. Gaussian marginals of convex bodies with symmetries. Beiträge Algebra Geom. 50 (2009) no. 1, pp. 101118.

[18] Rinott, Y.; Rotar, V. On coupling constructions and rates in the CLT for dependent summands with applications to the antivoter model and weighted $U$-statistics. Ann. Appl. Probab 7 (1997), no. 4.

[19] Stein, C. Approximate Computation of Expectations. Institute of Mathematical Statistics Lecture Notes-Monograph Series, 7, 1986.

[20] Stein, C.; Diaconis, P.; Holmes, S.; Reinert, G. Use of exchangeable pairs in the analysis of simulations. In Stein's method: expository lectures and applications, IMS Lecture Notes Monogr. Ser. 46, pp. 1-26, 2004. 